\title{
Monte Carlo homogenized limit analysis model for randomly assembled blocks in-plane loaded
}

\author{
Gabriele Milani $^{(1)}$, Paulo B. Lourenço ${ }^{(2)}$ \\ (1) Corresponding Author. Dipartimento di Ingegneria Strutturale (DIS), Politecnico di Milano, \\ Piazza Leonardo da Vinci 32, 20133 Milano, Italy. E-mail: milani@ stru.polimi.it. \\ ${ }^{(2)}$ ISISE, Department of Civil Engineering, School of Engineering, University of Minho, Azurém, \\ 4800-058 Guimarães, Portugal. E-mail: pbl@ civil.uminho.pt.
}

\begin{abstract}
A simple rigid-plastic homogenization model for the limit analysis of masonry walls in-plane loaded and constituted by the random assemblage of blocks with variable dimensions is proposed. In the model, blocks constituting a masonry wall are supposed infinitely resistant with a Gaussian distribution of height and length, whereas joints are reduced to interfaces with frictional behavior and limited tensile and compressive strength. Block by block, a representative element of volume (REV) is considered, constituted by a central block interconnected with its neighbors by means of rigid-plastic interfaces. The model is characterized by a few material parameters, is numerically inexpensive and very stable. A sub-class of elementary deformation modes is a-priori chosen in the REV, mimicking typical failures due to joints cracking and crushing. Masonry strength domains are obtained equating the power dissipated in the heterogeneous model with the power dissipated by a fictitious homogeneous macroscopic plate. Due to the inexpensiveness of the approach proposed, Monte Carlo simulations can be repeated on the REV in order to have a stochastic estimation of inplane masonry strength at different orientations of the bed joints with respect to external loads accounting for the geometrical statistical variability of blocks dimensions. Two cases are discussed, the former consisting on full stochastic REV assemblages (obtained considering a random variability of both blocks height an length) and the latter assuming the presence of a horizontal alignment along bed joints, i.e. allowing blocks height variability only row by row. The case of deterministic blocks height (quasi-periodic texture) can be obtained as a subclass of this latter case. Masonry homogenized failure surfaces are finally implemented in an upper bound FE limit analysis code for the analysis at collapse of entire walls in-plane loaded.

Two cases of engineering practice, consisting on the prediction of the failure load of a deep beam and a shear wall arranged with random texture are critically discussed. In particular, homogenization results are compared with those provided by a heterogeneous approach. Good agreement is found both on the failure mechanism and on the distribution of the collapse load.
\end{abstract}

Keywords: Masonry, in-plane loads, homogenization, random pattern, Monte Carlo simulations. 


\section{Introduction}

Masonry walls constituted by random assemblages of blocks and stones of variable dimensions, Figure 1, are very common in existing historical buildings. Therefore, the prediction of their ultimate strength under in-plane actions is a key issue for practitioners involved in the safety assessment of existing masonry structures.

At present, a key problem in the development of accurate stress analyses for random assemblages of blocks is the definition and the use of suitable material constitutive laws for masonry.

Micro-modeling, e.g. [1]-[4], considers units and mortar joints separately, characterizing them through different constitutive laws. Blocks and mortar mechanical properties adopted are usually obtained through experimental tests conducted on the single materials (compressive test, tension test, bending test, etc.). This approach is, of course, the most indicated for a detailed description of irregular masonry, but leads to structural analyses characterized by great computational effort, requiring several finite elements for a separate discretization of joints and blocks, even for small specimens. For the aforementioned limitations, micro-modeling can be successfully adopted only for walls of small dimensions (e.g. Lourenço and Rots [1], Lotfi and Shing [4]).

Macro-models, e.g. [5]-[6], substitute the heterogeneous material with a fictitious anisotropic homogeneous one, thus needing much less time when used in complex non linear analyses, but requiring a calibration of the model with expensive experimental data fittings.

Homogenization [7]-[11] appears a good compromise between micro and macro-modeling, as it allows to derive in a rational way stress-strain relationships for masonry, accounting in a suitable manner for the mechanical properties of each constituent material only at a cell level. Moreover, it may lead to effective models, with reduced computational effort for real scale simulations [7]-[11]. Stochastic homogenization concepts have been recently applied to random blocks assemblages both in the elastic and inelastic range [12]-[15], using suitable representative elements of volume (REVs) to describe the overall behavior of the wall under consideration.

In all cases where the periodicity of the structure is strictly not respected, it can be demonstrated [14]-[16] that a boundary value problem where periodic conditions are suitably relaxed has to be solved on the unit cell, in order to have a numerical estimation of the macroscopic inelastic properties to be used at a structural level. It is very straightforward to deduce that the geometrical variability of the REV, due to the variable geometry of the blocks, requires a statistical treatment of resultant (output) average masonry mechanical properties obtained through homogenization, since masonry strength strongly depends on the effective geometry of the REV considered. Therefore, a 
full set of Monte Carlo simulations are needed to have an estimation of mechanical properties distributions in case of both in- and out-of-plane loads.

In recent papers, e.g. [14]-[16], the effect of the stochastic variability of the blocks length on the inand out-of-plane elastic response of a periodic masonry wall has been studied. In particular, starting from a periodic running bond pattern consisting of rigid blocks with elastic interfaces, a random perturbation on the horizontal positions of the vertical interfaces between the blocks was introduced. Here, a similar compatible model constituted by a REV with a central block interconnected with neighboring blocks is studied, but (a) generalizing the problems in the case of stochastic variability of both height and length of the blocks and (b) assuming a rigid-plastic behavior for the constituent materials in order to have a prediction of masonry macroscopic behavior at failure.

As well known, limit analysis has been widely used for the study of masonry structures, see for instance [17]-[21], because it requires only a reduced number of material parameters, providing limit multipliers of loads, failure mechanisms and, at least on critical sections, the stress distribution at collapse. In this framework, with the aim of reproducing the behavior of masonry panels constituted by the random assemblage of blocks with variable dimensions, a mesoscopic compatible identification model is proposed.

Following what was presented in the case of regular assemblages of blocks in [22] and [23], joints are reduced to interfaces with a cohesive associated frictional behavior with limited tensile and compressive strength and blocks are supposed infinitely resistant. It is worth underlining here that frictional phenomena may require the adoption of non-associated flow rules for the constituent materials (see for instance [18], [19] and [24]). On the other hand, it has been demonstrated that even simple associated limit analyses (de Buhan and de Felice [17]) are able to provide reliable results, especially when failure mechanisms are mainly due to joints tensile cracking (e.g. [20]).

The present study concerns a random assemblage of blocks with variable height and length following a predetermined random distribution. In this way, the horizontal position of vertical joints as well as the vertical position of horizontal joints is statistically variable.

Since the case under consideration is in practice an assemblage of regular blocks with variable dimensions, it can be argued that the masonry skeleton may be represented by a 3D discrete system of blocks interacting through interfaces (the mortar joints). Modeling the REV as a molecular skeleton allows to strongly reduce degrees of freedom and therefore permits to perform full Monte Carlo simulations in case of geometrical stochastic variability of the geometry.

Within this simple model, a full description of the masonry material can be given considering a representative volume constituted by a generic block interconnected with its neighbors. A sub-class 
of possible elementary deformation modes (mimicking horizontal and vertical stretching, a pure shear deformation, pure bending and torsion along material axes) acting in the unit cell is a priori chosen in order to describe joints cracking under normal and tangential actions. Then, power dissipated in the discrete model is equated to that dissipated in a continuum macroscopic 2D equivalent plate. Such identification is based on a simple correspondence between motions in the 3D discrete model and the continuum. Since internal dissipation can take place only at the interface between blocks, a simple constrained minimization problem in a few variables is obtained. Macroscopic masonry failure surfaces are numerically evaluated as a function of the macroscopic in-plane actions (shear and normal actions). Here it is worth noting that, despite the fact that in this paper only in-plane actions are considered, the model is sufficiently general to analyze also random assemblages of blocks subjected to out-of-plane loads.

Due to the inexpensiveness of the approach proposed, large Monte Carlo simulations are repeated at a cell level, generating automatically a number of different REVs, sampling each time at random height and length of the blocks constituting the REV.

At a fixed geometry (i.e. within a single Monte Carlo sampled REV), in-plane masonry strength at different orientations of the bed joints with respect to external loads are numerically evaluated, solving for each orientation of the joint, several different linear programming problems, each optimization problem corresponding to a fixed direction of the load dependent from the load multiplier in the homogenized stress-space.

Two cases are discussed, the former consisting on REV assemblages without a preferential horizontal alignment, the latter assuming continuous horizontal joints. Afterwards, masonry homogenized failure surfaces are implemented in an upper bound FE limit analysis code for the analysis at collapse of in-plane loaded structural elements.

Two examples of engineering interest, consisting respectively on the prediction of the failure load of a deep beam and a shear wall arranged with random texture are critically discussed and compared with expensive heterogeneous approaches. The deep beam is selected as bending strength is significantly depending on the arrangement of the internal masonry structure, in the usual case of steeped cracks, and the shear wall is a typical validation structural element in the case of masonry structures. Good agreement is found at structural level between the models, meaning that the homogenization model proposed may be a valuable tool for limit analysis of masonry structures constituted by random assemblages of blocks. 


\section{Masonry homogenized failure surfaces: a model with infinitely resistant blocks and plastic mortar interfaces}

In this section, a procedure to obtain a general plate model (i.e. suitable both for in- and out-ofplane loaded panels) and based on a correspondence between equivalent class of motions in a 3D discrete blocks system and a 2D Cauchy continuum is presented. The two models are described separately and then an equivalence procedure between the kinematic descriptors in the two systems is performed, in order to study masonry as a 2D homogeneous continuum. First of all, the 3D model constituted by rigid infinitely resistant blocks interconnected by plastic mortar interfaces is introduced. The kinematic variables involved in the optimization problem used to find homogenized failure surfaces are only blocks velocities and rotation rates and plastic multipliers of the interfaces. While the procedure is general and can be applied also for out-of-plane load, here only the in-plane behavior is investigated.

After a brief recall of the basic features of the 3D discrete model, a transition between a discrete 3D block model and a 2D continuum model is presented. This is based on a simplified homogenization approach, where the discrete representative element of volume is connected to the 2D model by means of the assumption that the power dissipated in both models is identical. It is worth noting that the formulation of the model does not impose a field local solution as, for instance, occurs using standard homogenization procedures, but imposes only a kinematic correspondence between motions. This assumption implies that the obtained solution is kinematically admissible and, hence, an upper bound of the actual strength domains may be obtained with the model proposed.

\subsection{An automatic procedure to obtain a REV constituted by the random assemblage of blocks}

In order to repeat large scale Monte Carlo simulations on different REVs, an automatic routine of generation of REVs with blocks of different dimensions is needed.

In the present paper, we consider two different block assemblages, as schematically depicted in Figure 1 and Figure 2. The first distribution, here denoted as Case I, concerns the random assemblage of blocks with variable height and length. The second case (Case II) is again a distribution with variable length and height of blocks, but with a horizontal alignment of blocks along the bed joint. An important sub-class of problems is obtained from Case II when the height is a deterministic variable (quasi periodic assemblage).

Let $C^{B}$ be the position of the centroid of the generic block $B_{i j}$ in the 3D Euclidean space. Let $\mathbf{e}_{1}$ $\mathbf{e}_{2}-\mathbf{e}_{3}$ indicate the global frame of reference with $\mathbf{e}_{1}$ indicating horizontal direction, $\mathbf{e}_{2}$ indicating 
vertical direction and $\mathbf{e}_{3}$ indicating the direction normal to the wall, Figure 3 and Figure $4 . i$ and $j$ indices assume integer values indicating the position of the blocks with respect to the central blocks. For instance, $i=1$ and $j=1$ indicate the first block positioned on the right with respect to the central block. Subsequent blocks are disposed with one interface common to the central block in counterclockwise order.

More in detail, the random REV is built block by block starting from the central block by introducing a random perturbation on horizontal and vertical position of blocks centroids neighboring central block and assuming that blocks length and height are stochastic variables with assigned distribution, i.e.:

$$
\begin{aligned}
& x_{B_{i, j}}=x_{B_{i-1}}+\delta_{1} \frac{L_{B_{i-1}}}{2}+\delta_{2} \frac{\tilde{L}_{B_{i}}}{2} \\
& y_{B_{i, j}}=y_{B_{i-1}}+\gamma_{1} \frac{H_{B_{i-1}}}{2}+\gamma_{2} \frac{\tilde{H}_{B_{i}}}{2}
\end{aligned}
$$

Where $\tilde{L}$ and $\tilde{H}$ are sampled values of blocks length and height from normal distribution with mean values $\bar{L}$ and $\bar{H}$ and standard deviations $\sigma_{L}$ and $\sigma_{H}$, respectively. The parameters $\delta_{1,2}$ and $\gamma_{1,2}$ suitably assume values equal to $-1,0,1$ following a counterclockwise disposition.

Depending on the choice of parameters $\delta_{1,2}$ and $\gamma_{1,2}$, two different classes of problems have been analyzed, namely a disposition with no alignment along the horizontal and vertical direction, as shown in Figure 2-a, or a preferential disposition preserving the bed joint direction, as depicted in Figure 2-b.

\subsection{Heterogeneous model}

The heterogeneous model is represented by the random assemblage of infinitely resistant blocks connected by mortar joints reduced to interfaces with rigid-plastic behavior (Figure 3). The motion of a generic block $A$ may be described as a function of its center velocity $\mathbf{v}^{C_{A}}=\left[\begin{array}{lll}v_{x x}^{C_{A}} & v_{y y}^{C_{A}} & v_{z z}^{C_{A}}\end{array}\right]^{T}$ and its rotation rates $\boldsymbol{\Phi}^{A}=\left[\begin{array}{lll}\Phi_{x x}^{A} & \Phi_{y y}^{A} & \Phi_{z z}^{A}\end{array}\right]^{T}$. Starting from this assumption, the motions of all the blocks in contact to block $A$ may be described. Hence, to describe the kinematic model it suffices to take into consideration the interaction of a generic couple of blocks, $(A, B)$.

Let the point $P$ (local coordinates $\xi_{1}, \xi_{2}$ ) be a generic point on the interface $I$ between $A$ and $B$, as illustrated in Figure 5. Since $P \in I$ belongs respectively to $A$ and $B$ (where $I$ indicates the common interface between the two blocks), the following relations can be written: 
$\mathbf{v}^{A}(P)=\mathbf{v}^{C^{A}}+\mathbf{M}\left(\boldsymbol{\Phi}^{A}\right)\left(P-\mathbf{C}^{A}\right)$

$\mathbf{v}^{B}(P)=\mathbf{v}^{C^{B}}+\mathbf{M}\left(\boldsymbol{\Phi}^{B}\right)\left(P-\mathbf{C}^{B}\right)$

Here, $\mathbf{v}^{A}(P)\left(\mathbf{v}^{B}(P)\right)$ is the velocity of point $P$, which is considered belonging to block $A(B)$, and $\mathbf{M}(\mathbf{\Phi})$ is the following $3 \times 3$ skew matrix:

$$
\mathbf{M}(\boldsymbol{\Phi})=\left[\begin{array}{ccc}
0 & -\Phi_{z z} & \Phi_{y y} \\
\Phi_{z z} & 0 & -\Phi_{x x} \\
-\Phi_{y y} & \Phi_{x x} & 0
\end{array}\right]
$$

In equation ( 2 ), the position of point $P$ is evaluated with respect to a local frame $\left(\xi_{1} \xi_{2}\right)$ with origin on the centroid on the interface, see Figure 5. It is worth mentioning that the kinematic model here proposed is restricted to small rotation rates. The jump of velocity $[\mathbf{v}(P)]$ between blocks $A$ and $B$ in a point $\xi \in I$ is expressed by:

$$
\lfloor\mathbf{v}(P)\rfloor=\mathbf{v}^{B}(P)-\mathbf{v}^{A}(P)=\mathbf{v}^{C^{A}}-\mathbf{v}^{C^{B}}+\mathbf{M}\left(\boldsymbol{\Phi}^{A}\right)\left(P-\mathbf{C}^{A}\right)-\mathbf{M}\left(\boldsymbol{\Phi}^{B}\right)\left(P-\mathbf{C}^{B}\right)
$$

and the power dissipated at the interface $I$ can be written as:

$\pi^{I}=\int_{I}\left[\mathbf{t}^{A}(P) \cdot \mathbf{v}^{A}(P)+\mathbf{t}^{B}(P) \cdot \mathbf{v}^{B}(P)\right] d S=\int_{I} \mathbf{t}^{A}(P) \cdot[\mathbf{v}(P)] d S$

where $\mathbf{t}^{A}(P)=\left[\begin{array}{lll}\tau_{13}(P) & \tau_{23}(P) & \sigma_{33}(P)\end{array}\right]^{T}\left(\mathbf{t}^{B}(P)\right.$ is the stress vector acting at $\boldsymbol{\xi}$ on block $A(B)$, see Figure 5, with $\mathbf{t}^{A}(P)=-\mathbf{t}^{B}(P)$ ).

Obviously, power dissipated in the whole REV is simply the sum of power dissipated on all interfaces, i.e.

written as:

$\pi=\sum_{I=1}^{n^{I}} \pi^{I}$

with $n^{I}$ total number of interfaces in REV. Here it is worth noting that both the number of joints (horizontal and vertical) and the geometry of the interfaces in the REV varies case by case. At a fixed REV sampled with the Monte Carlo approach proposed, in the numerical model proposed, a sub-routine allows to store in a suitable database nodes and connectivity matrix of each interface automatically.

\subsection{Identification of the 3D blocky model with a 2D continuum}

Without loss of generality, for the most general case of REVs loaded in- and out-of-plane, a standard 2D Cauchy continuum, identified by its middle plane $S$ of normal $\mathbf{e}_{3}$ (Figure 4), is assumed as an equivalent plate homogenized model. The velocity field of a point $\mathbf{P}$ (coordinates 
$\left[\begin{array}{lll}x_{1}^{P} & x_{2}^{P} & x_{3}^{P}\end{array}\right]$ ) belonging to the equivalent continuum plate is given by fields $\mathbf{w}(\mathbf{x})$ (components $w_{1}$, $w_{2}$ and $w_{3}$ ) and $\boldsymbol{\Psi}(\mathbf{x})$ (components $\Psi_{1}$ and $\Psi_{2}$ ), representing respectively the velocity and rotations rates of the plate in correspondence of the point $\mathbf{x}=\left[\begin{array}{lll}x_{1}^{P} & x_{2}^{P} & 0\end{array}\right]$ laying in the middle plane of the plate.

The stored energy density in the equivalent plate model is:

$$
\pi=\left[\begin{array}{lll}
N_{11} & N_{12} & N_{22}
\end{array}\right]\left[\begin{array}{c}
\dot{E}_{11} \\
\dot{E}_{12}+\dot{E}_{21} \\
\dot{E}_{22}
\end{array}\right]+\left[\begin{array}{ll}
T_{13} & T_{23}
\end{array}\right]\left[\begin{array}{c}
\dot{\gamma}_{13} \\
\dot{\gamma}_{23}
\end{array}\right]+\left[\begin{array}{lll}
M_{11} & M_{12} & M_{22}
\end{array}\right]\left[\begin{array}{c}
\dot{\chi}_{11} \\
\dot{\chi}_{12}+\dot{\chi}_{21} \\
\dot{\chi}_{22}
\end{array}\right]
$$

Where the symbols in equation ( 7 ) have the following meaning:

$-\dot{\mathbf{E}}=\left[\begin{array}{c}\dot{E}_{11} \\ \dot{E}_{12}+\dot{E}_{21} \\ \dot{E}_{22}\end{array}\right]=\frac{1}{t} \int_{-t / 2}^{t / 2}\left[\begin{array}{c}\partial w_{1} / \partial x_{1}-x_{3} \partial \Psi_{1} / \partial x_{1} \\ \partial w_{2} / \partial x_{1}+\partial w_{1} / \partial x_{2}-x_{3}\left(\partial \Psi_{1} / \partial x_{2}+\partial \Psi_{2} / \partial x_{1}\right) \\ \partial w_{2} / \partial x_{2}-x_{3} \partial \Psi_{2} / \partial x_{2}\end{array}\right] d x_{3}$ is the in-plane strain rate vector, assuming $t$ as the masonry thickness, $\mathbf{w}=\left[\begin{array}{lll}w_{1} & w_{2} & w_{3}\end{array}\right]^{T}$ the velocity field in the continuous model (local frame of reference) and $\boldsymbol{\Psi}=\left[\begin{array}{lll}\Psi_{1} & \Psi_{2} & \Psi_{3}\end{array}\right]^{T}$ the rotation rate field;

$-\dot{\gamma}=\left[\begin{array}{c}\dot{\gamma}_{13} \\ \dot{\gamma}_{23}\end{array}\right]=\frac{1}{t} \int_{-t / 2}^{t / 2}\left[\begin{array}{l}\partial w_{3} / \partial x_{1}+\partial w_{1} / \partial x_{3} \\ \partial w_{3} / \partial x_{2}+\partial w_{2} / \partial x_{3}\end{array}\right] d x_{3}$ is the shear strain rate;

$-\dot{\chi}=\left[\begin{array}{c}\dot{\chi}_{11} \\ \dot{\chi}_{12}+\dot{\chi}_{21} \\ \dot{\chi}_{22}\end{array}\right]=\frac{1}{t} \int_{-t / 2}^{t / 2}\left[\begin{array}{c}\partial \Psi_{1} / \partial x_{1} \\ \partial \Psi_{2} / \partial x_{1}+\partial \Psi_{1} / \partial x_{2} \\ \partial \Psi_{2} / \partial x_{2}\end{array}\right] d x_{3}$ is the curvature rate vector;

- $\mathbf{M}=\left[\begin{array}{lll}M_{11} & M_{12} & M_{22}\end{array}\right]^{T}$ is the homogenized moments vector, with $M_{11}$ and $M_{22}$ indicating bending and $M_{12}$ torsion;

- $\mathbf{T}=\left[\begin{array}{ll}T_{13} & T_{23}\end{array}\right]^{T}$ is the homogenized out-of-plane shear vector;

- $\mathbf{N}=\left[\begin{array}{lll}N_{11} & N_{12} & N_{22}\end{array}\right]^{T}$ is the homogenized membrane actions vector.

With the aim of limiting the analyses to in-plane actions, we assume in the continuous model that $\mathbf{M}=\mathbf{O}_{3}, \dot{\chi}=\mathbf{O}_{3}, \mathbf{T}=\mathbf{O}_{2}$ and $\dot{\gamma}=\mathbf{O}_{2}$, where $\mathbf{O}_{3}$ and $\mathbf{O}_{2}$ are $3 \times 1$ and $2 \times 1$ vector of all zeros respectively.

\subsection{Simplified homogenization}

The aim of the homogenized approach proposed is to substitute the heterogeneous material with a homogeneous equivalent 2D model, see Figure 6, where the term "equivalent" refers to an equivalence in terms of internal power dissipation. With this target in mind, a simple but effective compatible identification model is proposed, where we assign a sub class of possible deformation 
modes and we constraint that the power dissipated by blocks assemblage, equation ( 5 ), is equal to the power dissipated by the equivalent model, equation ( 7 ). For this purpose, fields $\mathbf{w}(\mathbf{x})$ and $\Psi(\mathbf{x})$ should be a priori chosen in a pre-assigned class of elementary deformations in the unit cell, corresponding to actual failure mechanisms occurring in presence of infinitely resistant blocks with weak joints reduced to interfaces. From a practical point of view, fields $\mathbf{w}(\mathbf{x})$ and $\Psi(\mathbf{x})$, corresponding to each sub-class of regular motions, are obtained assuming alternatively one component of vector $\dot{\mathbf{E}}$ unitary and setting all the other components equal to zero, subsequently choosing the most simple polynomial expressions for $\mathbf{w}(\mathbf{x})$ and $\Psi(\mathbf{x})$ that comply with equation ( 7 ). Once fields $\mathbf{w}(\mathbf{x})$ and $\boldsymbol{\Psi}(\mathbf{x})$ are known, rotations rates and velocities of each block belonging to the REV in the heterogeneous model are determined assuming point $\mathbf{x}$ as the centroid of the block under consideration.

For instance, when only $\dot{E}_{11} \neq 0$ is applied on the REV, an obvious choice for $\mathbf{w}(\mathbf{x})$ and $\Psi(\mathbf{x})$ fields is:

$$
\begin{aligned}
& \Psi_{1}=\Psi_{2}=\Psi_{3}=0 \\
& w_{1}=\dot{E}_{11} x_{1} \\
& w_{2}=0 \\
& w_{3}=0
\end{aligned}
$$

Equation ( 8 ) allows to directly determine velocities and rotations of each block, provided that the coordinates of the respective centroid are introduced in ( 8 ).

For instance, Figure 7-a shows the effect on a randomly assembled REV of a homogeneous deformation $\dot{E}_{11} \neq 0$ with all the other strain measures set to zero. It must be noted that both head and bed joints are involved in the dissipation induced by this deformation. Figure 7-b shows the effect on the block-work of a homogeneous deformation in which $\dot{E}_{12}+\dot{E}_{21} \neq 0$ (shear mode) and all the other strain measures are set to zero, whereas Figure 7-c shows the effect of a vertical in-plane homogeneous deformation $\dot{E}_{22} \neq 0$. Differently from a regular assemblage of blocks, for an irregular texture it is interesting to notice that both head and bed joints may contribute to the overall strength of the continuous material. On the contrary, when dealing with quasi-periodic masonry (i.e. with a horizontal alignment along bed joints), failure due to a macroscopic deformation $\dot{E}_{22} \neq 0$ involves only the bed joints, similarly to the regular running bond case. Similar considerations may be repeated for the out-of-plane deformation modes, depicted from Figure 7-d to -f. The irregular disposition of blocks causes, analogously to the in-plane case, bending moment and torsion both in the head and bed joints. 


\subsection{Stochastic masonry failure surfaces}

In this section, following the original formulation provided by Suquet [25], a general numerical procedure for obtaining macroscopic masonry failure surfaces is presented. Both static and kinematic theorems of limit analysis can be used for this purpose. In this framework, it is worth noting that several different models have been presented in the literature for the evaluation of inplane (e.g. Milani et al. [20]) and out-of-plane masonry failure surfaces (Sab [26], Cecchi and Milani [22]).

One of the basic assumptions of this approach is the utilization of associated flow rules for the constituent materials. Nevertheless, it is worth mentioning that sliding occurs in mortar joints with almost zero dilatancy, with typical non-associativity. The violation of one of the hypothesis of classic limit analysis [18][19], implies that the uniqueness of the ultimate load may be lost and a multiplicity of solutions can exist, see Begg and Fishwick [24]. On the contrary, the assumption of associated flow rules ensures the uniqueness of the ultimate load factor and leads to simple optimization problems which can be handled easily with linear programming (LP) packages.

A failure criterion $\phi=\phi(\boldsymbol{\sigma})$ for the joints must be incorporated. The basic failure modes for masonry walls with weak mortar are a mixing of sliding along the joints (a), cracking of the joints (b) and compressive masonry crushing (c). These modes can be well reproduced adopting a MohrCoulomb failure criterion combined with a tension cut-off and a cap in compression, see Figure 8, as suggested by Lourenço and Rots [1].

Aiming at treating the problem in the framework of linear programming, within each interface $I$ of area $A^{I}$, a piecewise linear approximation of the failure surface $\phi=\phi(\boldsymbol{\sigma})$ is adopted, constituted by $n_{\text {lin }}$ planes of equation $\mathbf{A}_{i}^{I^{T}} \boldsymbol{\sigma}=c_{i}^{I} \quad 1 \leq i \leq n_{\text {lin }}$, where $\boldsymbol{\sigma}=\left[\begin{array}{lll}\sigma_{33} & \tau_{13} & \tau_{23}\end{array}\right], \sigma_{33}$ is the normal stress on the interface and $\tau_{13}$ and $\tau_{23}$ are tangential stresses along two assigned perpendicular directions $\left(A_{i}^{1 I} \sigma_{33}+A_{i}^{2 I} \tau_{13}+A_{i}^{3 I} \tau_{23}=c_{i}^{I}\right.$ is the $i$-th linearization plane of the interface $I$, with $\left.\mathbf{A}_{i}^{I^{T}}=\left[\begin{array}{lll}A_{i}^{1 I} & A_{i}^{2 I} & A_{i}^{3 I}\end{array}\right]\right)$, Figure 5 and Figure 8.

The jump of velocity on interfaces varies linearly in the discrete model, equation ( 4 ). Thus, for each interface, only $3 \cdot n_{\text {lin }}$ independent plastic multiplier rates have to be introduced as optimization variables. Furthermore, for each interface $I$ between contiguous blocks, the following equality constraints between plastic multiplier rates $\dot{\lambda}_{i}^{I}\left(\xi_{1}, \xi_{2}\right)$ and jump of velocity $\left[\mathbf{v}\left(\xi_{1}, \xi_{2}\right)\right]$ on the interface must be imposed:

$$
\left[\mathbf{v}\left(\xi_{1}, \xi_{2}\right)\right]=\sum_{i=1}^{n_{\text {lin }}} \dot{\lambda}_{i}^{I}\left(\xi_{1}, \xi_{2}\right) \frac{\partial \phi}{\partial \boldsymbol{\sigma}}
$$


where:

- $\xi=\left(\xi_{1}, \xi_{2}\right)$ is a local frame of reference laying on the interface plane and with axis $\xi_{3}$ orthogonal to the interface plane, Figure 8;

- $\left[\mathbf{v}\left(\xi_{1}, \xi_{2}\right)\right]=\left[\begin{array}{lll}\Delta v_{33} & \Delta v_{13} & \Delta v_{23}\end{array}\right]^{T}$ is the jump of velocity field (linear in $\left(\xi_{1}, \xi_{2}\right)$ ) on the $I$-th interface and $\Delta v_{i j}$ corresponds to the jump along the direction $j$.

- $\dot{\lambda}_{i}^{I}\left(\xi_{1}, \xi_{2}\right)$ is the $i$-th plastic multiplier rate field (linear in $\left(\xi_{1}, \xi_{2}\right)$ ) of the interface $I$, associated to the $i$-th linearization plane of the failure surface.

It is worth noting that, in order to satisfy equation ( 9 ) for each point of the interface $I$, nine equality constraints for each interface have to be imposed, which corresponds to evaluating ( 9 ) in three different positions $P_{k}=\left(\xi_{1}^{P_{k}}, \xi_{2}^{P_{k}}\right)$ on the interface $I$ as follows:

$$
\left[\mathbf{v}\left(\xi_{1}^{P_{k}}, \xi_{2}^{P_{k}}\right)\right]=\sum_{i=1}^{n_{\text {lin }}} \dot{\lambda}_{i}^{I}\left(\xi_{1}^{P_{k}}, \xi_{2}^{P_{k}}\right) \frac{\partial \phi}{\partial \boldsymbol{\sigma}} \quad k=1,2,3
$$

Here, $\dot{\lambda}_{i}^{I}\left(\xi_{1}^{P_{k}}, \xi_{2}^{P_{k}}\right)$ is the is $i$-th plastic multiplier rate of the interface $I$ corresponding to $P_{k}=\left(\xi_{1}^{P_{k}}, \xi_{2}^{P_{k}}\right)$.

From the previous equations, the internal power dissipated on the $I$-th interface can be written as:

$$
\pi_{\text {int }}^{I}=\int_{A^{I}}[\mathbf{v}]^{T} \boldsymbol{\sigma} d A^{I}=\int_{A^{I}} \sum_{i=1}^{n_{\text {lin }}} \dot{\lambda}_{i}^{I}\left(\xi_{1}, \xi_{2}\right)\left[\frac{\partial \phi}{\partial \boldsymbol{\sigma}}\right]^{T} \boldsymbol{\sigma} d A^{I}=\frac{1}{4} \sum_{i=1}^{n_{\text {lin }}} c_{i}^{I} \sum_{k=1}^{4} \dot{\lambda}_{i}^{I}\left(\xi_{1}^{P_{k}}, \xi_{2}^{P_{k}}\right) A^{I}
$$

It is worth noting that in equation ( 11 ) only three of the four plastic multipliers are linearly independent, whereas $k=4$ depends linearly on $k=1,2,3$ (the plastic multiplier field is linear on the interface).

The external power dissipated can be written as $\pi_{e x t}=\left(\Sigma_{0}^{T}+\lambda \Sigma_{1}^{T}\right) \mathbf{D}$, where $\Sigma_{0}$ is the vector of dead loads, $\lambda$ is the load multiplier, $\Sigma_{1}^{T}$ is the unitary vector of loads dependent on the load multiplier (i.e. the optimization direction in the space of macroscopic stresses) and $\mathbf{D}$ is the vector of macroscopic kinematic descriptors. D collects in-plane deformation rates $\left(\dot{E}_{11} 0.5\left(\dot{E}_{12}+\dot{E}_{21}\right) \dot{E}_{22}\right)$, Kirchhoff-Love out-of-plane curvature rates $\left(\dot{\chi}_{11}\left(\dot{\chi}_{12}+\dot{\chi}_{21}\right) / 2 \dot{\chi}_{22}\right)$ and out-of-plane shear deformation rates $\left(\dot{\gamma}_{13}\right.$ and $\left.\dot{\gamma}_{23}\right)$. As the amplitude of the failure mechanism is arbitrary, a further normalization condition $\boldsymbol{\Sigma}_{1}^{T} \mathbf{D}=1$ is usually introduced. Hence, the external power becomes linear in $\mathbf{D}$ and $\lambda$ and can be written as follows $\pi_{e x t}=\boldsymbol{\Sigma}_{0}^{T} \mathbf{D}+\lambda$.

From the above considerations, optimization variables necessary to determine masonry homogenized strength domain are respectively the vector of macroscopic kinematic descriptors D and the vector of assembled plastic multiplier rates $\dot{\lambda}^{I}$ at each mortar interface. 
From equations ( 8 ) and ( 4 ), a further set of linear equality constraints has to be imposed at each interface $I$, involving vector $\mathbf{D}$ and jump of displacements field $\left[\mathbf{v}\left(\xi_{1}, \xi_{2}\right)\right]$ :

$$
\left[\mathbf{v}\left(\xi_{1}, \xi_{2}\right)\right]=\mathbf{G}^{I}\left(\xi_{1}, \xi_{2}\right) \mathbf{D}
$$

where $\mathbf{G}^{I}\left(\xi_{1}, \xi_{2}\right)$ is a $3 \times 8$ matrix that depends only on the geometry of the interface under consideration. It is interesting to notice that, from equations ( 10 ) and ( 12 ), the jump of velocities $\left[\mathbf{v}\left(\xi_{1}, \xi_{2}\right)\right]$ does not enter as optimization variable in the optimization problem at a cell level, being $\mathbf{G}^{I}\left(P_{k}\right) \mathbf{D}=\left[\mathbf{v}\left(P_{k}\right)\right]=\sum_{i=1}^{n_{\text {lin }}} \dot{\lambda}_{i}^{I}\left(\xi_{1}^{P_{k}}, \xi_{2}^{P_{k}}\right) \frac{\partial \phi}{\partial \boldsymbol{\sigma}} \quad P_{k} \in I$. In particular, from equations ( 10$),(11),(12)$ and from the kinematic formulation of limit analysis, the following constrained minimization problem has to be solved to obtain masonry failure surfaces:

$$
\left\{\begin{array}{c}
\lambda=\min _{\hat{\mathbf{x}}=\left[\mathbf{D}, \lambda_{i}^{l}\left(P_{k}\right)\right]} \sum_{I=1}^{n^{I}} \pi_{\mathrm{int}}^{I}-\boldsymbol{\Sigma}_{0}^{T} \mathbf{D} \\
\boldsymbol{\Sigma}_{1}^{T} \mathbf{D}=1 \\
\mathbf{G}^{I}\left(P_{k}\right) \mathbf{D}=\left[\mathbf{v}\left(P_{k}\right)\right]=\sum_{i=1}^{n_{\text {lin }}} \dot{\lambda}_{i}^{I}\left(\xi_{1}^{P_{k}}, \xi_{2}^{P_{k}}\right) \frac{\partial \phi}{\partial \boldsymbol{\sigma}} \quad P_{k} \in I
\end{array}\right.
$$

Here, $n^{I}$ is the total number of interfaces considered and $\hat{\mathbf{x}}$ is the vector of total optimization unknowns. The linear programming problem ( 13 ) involves a relatively small number of optimization variables and therefore can be solved by means of simplex or interior point methods (vector $\hat{\mathbf{x}}$ of global unknowns collects only $3 \cdot n_{\text {lin }} \cdot n^{I}$ plastic multiplier rates and 8 macroscopic kinematic variables D). When it is required to investigate only masonry homogenized in-plane behavior, D is a vector of length three collecting in-plane macroscopic deformation rates ( $\dot{E}_{11}$, $0.5\left(\dot{E}_{12}+\dot{E}_{21}\right)$ and $\left.\dot{E}_{22}\right)$, whereas masonry macroscopic strength domain is a surface in the space of membrane actions $\left(N_{11}, N_{12}\right.$ and $\left.N_{22}\right)$. Obviously, the optimal value $\lambda$ obtained from ( 13 ) represents only a point on $\hat{\Phi}$, i.e. the intersection between surface $\hat{\Phi}$ and the direction unit vector $\Sigma_{1}$, see also Figure 9. Consequently, in order to obtain a reliable linear approximation of $\hat{\Phi}$ by means of Delaunay tessellations, the linear programming problem ( 13 ) has to be solved several times, each problem corresponding to a different choice for $\boldsymbol{\Sigma}_{1}$ direction.

Usually, masonry failure surface sections are represented assuming a fixed angle $\vartheta$ between the bed joint and the macroscopic horizontal action $\left(\sigma_{h h}\right)$. Such sections are obtained keeping $\vartheta$ fixed and varying point by point $\psi$ angle, defined as $\psi=\tan ^{-1} \sigma_{v v} / \sigma_{h h}$, where $\sigma_{v v}$ is the macroscopic vertical action. In this framework, vector $\boldsymbol{\Sigma}_{1}$ has the following form: 


$$
\begin{gathered}
\Sigma_{1,1}=\frac{1}{2}(\cos (\psi)(1+\cos (2 \vartheta))+\sin (\psi)(1-\cos (2 \vartheta))) \\
\Sigma_{1,2}=\frac{1}{2}(\cos (\psi)(1-\cos (2 \vartheta))+\sin (\psi)(1+\cos (2 \vartheta))) \\
\Sigma_{1,3}=\frac{1}{2}(\cos (\psi) \cos (2 \vartheta)-\sin (\psi) \cos (2 \vartheta)) \tan (2 \vartheta) \\
\Sigma_{1-4,5,6}=0
\end{gathered}
$$

As already pointed out, we limit here the study to in-plane actions, but the procedure is much more general and random out-of-plane failure surfaces will be presented in another contribution.

\subsection{Two meaningful application at a cell level}

Two meaningful applications at a cell level are here analyzed, the first consisting in a random assemblage of blocks with variable size (i.e. with length and height regarded as stochastic variables, without preferential lines in their disposition - Case I) and the second relying in a so-called quasi periodic disposition of blocks, i.e. where horizontal alignment along bed joints is preserved (Case II). In both cases, large scale Monte Carlo simulations are performed $(10,000)$ at fixed orientations $\vartheta$ of the central block with respect to the direction of horizontal membrane loads. Three different $\vartheta$ angles are considered, in analogy to Page [27] experimental results, namely $0^{\circ}, 22.5^{\circ}$ and $45^{\circ}$. For each orientation $\vartheta$ and at a fixed REV geometry (corresponding to a single Monte Carlo simulation), failure surface sections are obtained with the model proposed investigating 40 different $\psi$ angles equally stepped, thus requiring to solve iteratively a total of 1,200,000 optimization problems ( 13 ) for each example.

In both cases, due to the very limited number of variables involved in the simulations, the computational effort required to perform such large scale Monte Carlo simulations did not exceed 3,600 seconds for each $\vartheta$ section, meaning that the procedure proposed may be a valuable numerical tool for all practitioners interested to a reliable and fast estimation of collapse load distributions of random blocks assemblages.

\subsubsection{Case I: random assemblage through blocks with variable dimensions}

Let us consider a masonry wall arranged through rectangular blocks disposed in irregular texture (Case I) and mortar joints reduced to interfaces. This pattern typology is somewhat diffused in historical buildings (see Figure 1-a) and it is therefore of interest to determine typical REV behavior near failure when loaded in-plane. We assume for the blocks a stochastic normal distribution for length $\mathrm{L}$ and height $\mathrm{H}$, with mean values equal to 300 and $200 \mathrm{~mm}$ respectively for $\mathrm{L}$ and $\mathrm{H}$. Standard deviations are set equal to 80 and $60 \mathrm{~mm}$ respectively for length and height. Mechanical properties at failure adopted for the constituent materials are summarized in Table I. In particular, 
for mortar joints, a linearized Lourenço and Rots [1] failure criterion is adopted, whereas blocks are assumed infinitely resistant.

In Figure 10, $\sigma_{h h}-\sigma_{v v}$ masonry in-plane strength domains obtained with the model proposed sampling a total number of 10,000 different REVs and assuming $\vartheta=0^{\circ}$ are represented. The same results are replicated respectively in Figure 11 and Figure 12 for $\vartheta=22.5^{\circ}$ and $\vartheta=45^{\circ}$.

For the sake of completeness, in Figure 13 some typical failure mechanisms obtained at fixed $\psi$ and $\vartheta$ angles are reported for some sampled REV dispositions.

From an overall analysis of simulations results, the following remarks are worth noting:

1) The minimum envelope of the resultant homogenized failure surfaces is isotropic and obviously corresponds to a homogenized failure surface obtained supposing masonry constituted by mortar joints reduced to interfaces interconnected by blocks disposed in stack bond texture (i.e. with both vertical and horizontal alignment).

2) The possible non Gaussian behavior of the failure multipliers (see for instance Figure 12) at different $\psi$ angles of the resultant homogenized failure surface can be easily explained remembering that very different failure mechanisms are associated -at fixed $\psi$ angles- to very different failure multipliers. Therefore, it is straightforward to conclude that such distributions collect failure loads not necessarily connected between each other, because they are related to different failure mechanisms.

3) The very limited computation effort required to perform the simulations justifies the two steps procedure here proposed, consisting in collecting failure surfaces in a database to implement successively at a structural level for upper bound limit analyses of entire structures.

\subsubsection{Case II: random assemblage with preferential horizontal disposition}

A random assemblage of blocks with preferential horizontal disposition is studied as a second example. The same stochastic distribution of the previous example is assumed here for the block, but maintaining in this case the horizontal alignment fixed and staggering blocks along two contiguous rows. Mechanical properties at failure adopted for the constituent materials are the same of the previous example, see Table I.

In Figure 14, $\sigma_{h h}-\sigma_{v v}$ masonry in-plane strength domains obtained with the model proposed at fixed $\vartheta$ angle equal to $0^{\circ}$ and resulting from a large scale Monte Carlo set of simulations is shown. The same results are replicated respectively in Figure 15 and Figure 16 for $\vartheta=22.5^{\circ}$ and $\vartheta=45^{\circ}$. For the sake of completeness, in Figure 17 some typical failure mechanisms obtained at fixed $\psi$ and $\vartheta$ angles are reported for some sampled REV dispositions. 
From an overall analysis of simulations results, the following remarks are worth noting:

1) due to the horizontal alignment of the blocks forming a continuous bed joint, there is no variability of vertical strength. In fact, the REV always fails along the preferential plane of weakness formed by the bed joint.

2) the typical anisotropic behavior of masonry along the material axes is more marked with respect to the previous case. In Case I, the general major strength of the REV along horizontal direction is essentially due to the ratio between blocks length and height $>1$. This generates a small staggering of the blocks along two contiguous horizontal courses, contributing to an additional horizontal resistance. In this case, blocks staggering is a priori imposed in the disposition of the blocks and only the position of the vertical joints is stochastically perturbed, thus justifying the more marked anisotropic behavior of the REV.

\section{Structural level implementation}

A homogenized upper bound approach is utilized for the stochastic structural analysis at collapse of entire masonry walls. The formulation is based on a triangular discretization of 2D domains and on the introduction of discontinuities of the velocity field along the edges of adjacent triangles. For the homogenization model, also a mesh adaptation based on a sequential linear programming approach recently presented by Milani and Lourenço [28] is adopted, in order to obtain reliable evaluation of collapse loads even with very coarse meshes (thus allowing expensive Monte Carlo simulations at a relatively low computational cost).

For each element $E$, two velocity unknowns per node $i-u_{x x}^{i}$ and $u_{y y}^{i}$ (one horizontal and one vertical, see Figure 18-a) are introduced, so that the velocity field is linear inside an element, whereas the strain rate field is constant.

Jumps of velocities on interfaces are supposed to vary linearly. Hence, for each interface, four unknowns are introduced $\left(\Delta \mathbf{u}^{I}=\left[\Delta v_{1} \Delta u_{1} \Delta v_{2} \Delta u_{2}\right]^{T}\right)$, representing the normal $\left(\Delta v_{i}\right)$ and tangential $\left(\Delta u_{i}\right)$ jumps of velocities (with respect to the discontinuity direction) evaluated on nodes $i=1$ and $i=2$ of the interface (see Figure 18-b). For any pair of nodes on the interface between two adjacent triangles $(m)-(n)$, the tangential and normal velocity jumps can be written in terms of the Cartesian nodal velocities of elements $(m)-(n)$ (Sloan and Kleeman [29]), so that four linear equations in the form $\mathbf{A}_{11}^{e q} \mathbf{u}^{E m}+\mathbf{A}_{12}^{e q} \mathbf{u}^{E n}+\mathbf{A}_{13}^{e q} \Delta \mathbf{u}^{I}=\mathbf{0}$ can be written for each interface, where $\mathbf{u}^{E m}$ and $\mathbf{u}^{E n}$ are the $6 x 1$ vectors that collect velocities of elements $(m)$ and $(n)$ respectively.

For the continuum, three equality constrains representing the plastic flow in continuum (obeying an associated flow are introduced for each element: 


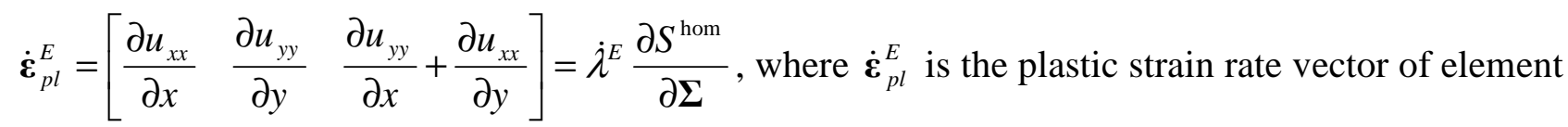
$E, \quad \dot{\lambda}^{E} \geq 0$ is the plastic multiplier and $S^{\text {hom }}$ is the (non) linear failure surface. A linear approximation (with $m$ planes) of the failure surface is at disposal in the form $S^{\text {hom }} \equiv \mathbf{A}^{\text {in }} \boldsymbol{\Sigma} \leq \mathbf{b}^{\text {in }}$; therefore three linear equality constraints per element can be written in the form $\mathbf{A}_{11}^{e q} \mathbf{u}^{E}+\mathbf{A}_{12}^{e q} \dot{\lambda}^{E}=\mathbf{0}$, where $\mathbf{u}^{E}$ is the vector of element velocities and $\dot{\lambda}^{E}$ is a $m x 1$ vector of plastic multiplier rates (one for each plane of the linearised failure surface).

For the interfaces, a 2D projection of the 3D failure surfaces is required, which depends on the orientation $\vartheta$ of the interface with respect to the horizontal direction. The general procedure suggested by Krabbenhoft et al. [30] is here adopted and the reader is referred there for further details.

Once the linearised domains for interfaces are provided by means of (6), the power dissipated on the discontinuities is computed introducing plastic multipliers for every interface $I$ as follows:

$$
\Delta \mathbf{u}^{I}(\xi)=\left[\begin{array}{l}
\Delta v(\xi) \\
\Delta u(\xi)
\end{array}\right]=\sum_{i=1}^{m} \dot{\lambda}_{I}^{(i)}(\xi) \nabla f_{\sigma}^{(i)}
$$

Where $\xi$ is the abscissa of $I, \nabla f_{\sigma}{ }^{(i)}$ are constant gradients for the failure surface (being $f^{(i)}$, the $i^{\text {th }}$ segment of the multi-linear failure surface), $\dot{\lambda}_{I}^{(i)}$ are the interface plastic multiplier rates, evaluated in correspondence of $\xi$, integrated along the infinitesimal thickness of interfaces and associated with the $i^{\text {th }}$ segment of the multi-linear failure surface, $\Delta v$ and $\Delta u$ have been already introduced and are respectively the normal and tangential jump of velocities on the discontinuities.

After some elementary assemblage operations, a simple linear programming problem is obtained, where the objective function consists in the minimization of the total internal power dissipated:

$$
\left\{\begin{array}{c}
\min \left\{\mathbf{C}_{E}^{T} \dot{\lambda}^{E, a s s}+\mathbf{C}_{I}^{T} \dot{\lambda}^{I, \text { ass }}\right\} \\
\text { such that }\left\{\begin{array}{l}
\mathbf{A}^{\text {eq }} \mathbf{U}=\mathbf{b}^{\text {eq }} \\
\dot{\boldsymbol{\lambda}}^{I, \text { ass }} \geq \mathbf{0} \\
\dot{\boldsymbol{\lambda}}^{E, \text { ass }} \geq \mathbf{0}
\end{array}\right.
\end{array}\right.
$$

where:

- $\mathbf{C}_{E}^{T}$ and $\mathbf{C}_{I}^{T}$ are the (assembled) right-hand sides of the inequalities that determine the linearised failure surface of the homogenized material respectively in the continuum and in the interfaces.

- $\mathbf{U}=\left[\begin{array}{llll}\mathbf{u} & \dot{\lambda}^{\text {E,ass }} & \Delta \mathbf{u}^{I, \text { ass }} & \dot{\lambda}^{I, \text { ass }}\end{array}\right\rfloor$ is the vector of global unknowns, which collects the vector of assembled nodal velocities ( $\mathbf{u})$, the vector of assembled element plastic multiplier rates $\left(\dot{\lambda}^{E, a s s}\right)$, the 
vector of assembled jump of velocities on interfaces $\left(\Delta \mathbf{u}^{I, a s s}\right)$ and the vector of assembled interface plastic multiplier rates $\left(\dot{\lambda}^{I, a s s}\right)$.

- $\mathbf{A}^{e q}$ is the overall constraints matrix and collects velocity boundary conditions, relations between velocity jumps on interfaces and elements velocities, constraints for plastic flow in velocity discontinuities and constraints for plastic flow in continuum.

It is worth noting that $\mathbf{C}_{E}^{T} \dot{\lambda}^{\text {Eass }}$ and $\mathbf{C}_{I}^{T} \dot{\lambda}^{\text {I, ass }}$ in the objective function represent respectively the total power dissipated in continuum and in interfaces.

When dealing with the homogenized approach, a mesh adaptation based on a sequential linear programming scheme (SLP, see the original formulation proposed by Milani and Lourenço [28]) is adopted in order (-a) to utilize very coarse meshes and hence to take advantage of the most important potential of homogenization (i.e. disregard blocks pattern at structural level) and (-b) to improve step by step the reliability of the failure load provided by a coarse mesh.

A full description of the SLP scheme adopted is available in [28]. Here, it is worth noting that, differently from the original formulation proposed in [28], (a) linear triangles are used instead of splines based elements and (b) plastic dissipation is allowed also inside triangular elements. Convergence is usually reached after a few iterations (5 to 15) and therefore the procedure is particularly suited to be extended to the Monte Carlo simulations here performed.

All the numerical simulations reported in the paper have been performed under Matlab 7.8.0 on a Windows Vista PC equipped with an Intel Core 2 Duo CPU @ 2 GHz and 4 GByte RAM. Here it is worth noting that, from a numerical point of view, a parallelization of the analyses through large clusters would be very beneficial for the problem at hand, since large scale Monte Carlo simulations would require much less time to be performed or, conversely, at fixed time, the number of simulations would increase drastically. However, a single PC commonly available in the market stock has been used having in mind that results should be replicated by any practitioner interested in a relatively fast stochastic analysis of real scale engineering structures.

\section{Deep beam subjected to a vertical concentrated load}

A masonry deep beam of dimensions $600 \times 200 \mathrm{~cm}$ arranged in random texture with blocks dimensions (mean values) equal to $300 \times 120 \mathrm{~mm}$ (length $\mathrm{x}$ width) and simply supported in correspondence of the first and last block of the first row of blocks starting from the bottom (see Figure 19) is considered. A vertical concentrated load is placed at the middle top of the wall and incremented until failure $\left(\mathrm{L}_{\mathrm{p}}=300 \mathrm{~mm}\right)$. The behavior at failure of the panel assuming for the blocks the dispositions shown in Figure 2 is extensively analyzed, with comparisons with alternative expensive heterogeneous approaches. In both models, a frictional failure surface with limited tensile 
strength and cap in compression is considered (Lourenço and Rots [1] failure criterion) for mortar joints. Mechanical properties assumed in the simulations are summarized in Table II.

\subsection{No horizontal alignment (Case I disposition): homogenized analyses}

A set of Monte Carlo simulations is performed on the structural model at hand, discussing the effect of changing the coefficient of variation (COV) of the height and the length of the blocks, assuming the wall arranged through the first disposition of blocks (Case I) discussed in the previous sections. Six different values of COV for $\mathrm{H}$ and $\mathrm{L}$ are investigated, respectively equal to 2.5-5-10-15-2025\%. At fixed values of $\mathrm{H}$ and L COVs, a set of 2,000 Monte Carlo simulations is performed, meaning that a total of 72,000 structural analyses are repeated for the problem under consideration. In Figure 20, results obtained from the numerical simulations are represented in terms of empirical cumulative distribution functions (ECDFs), at fixed length and height COVs. Mean values of the distributions estimated thorough the Monte Carlo simulations are also represented, along with the deterministic values obtained assuming a stack bond and a running bond disposition for the blocks, using respectively black, red and purple thick curves.

The following four key aspects are worth noting from the distributions, confirming that the procedure proposed is able to reproduce accurately -but at a fraction of the computational time needed by standard heterogeneous approaches- the behavior of irregular assemblages of blocks near failure:

1. When a small COV for both the height and the length of the blocks (upper left diagram of Figure 20) is assumed (e.g. 2.5\%), the behavior of the wall is closer to the stack bond situation. As can be seen from Figure 2-a, due to the adopted definition of geometry, Case I arrangement reduces to a stack bond disposition when $\mathrm{H}$ and L COVs tend to zero.

2. The mean failure load and standard deviation increase with the height $\mathrm{H}$ and length L COVs. Both parameters play an important role, with a slightly more relevant role on the length: for a L COV of $5 \%$ the mean failure loads are 107 and $123 \mathrm{kN}$, for a $\mathrm{H} \mathrm{COV}$ of $5 \%$ and $25 \%$ respectively; for a L COV of $25 \%$ the mean failure loads are 131 and $146 \mathrm{kN}$, for a $\mathrm{H} \mathrm{COV}$ of $5 \%$ and $25 \%$ respectively.

3. The limiting upper bound case of the running bond texture cannot be achieved with the increasing COVs, because the extra resistance due to the full staggering of the blocks is never reached. 


\subsection{Case II disposition: quasi periodic arrangement}

A second set of Monte Carlo simulations $(2,000)$ is performed assuming a quasi periodic disposition of the blocks, i.e. imposing that bricks height is constantly equal to the mean value and considering the second disposition of the blocks shown in Figure 2 (Case II). In order to validate the results obtained with the model proposed an expensive set of heterogeneous Monte Carlo simulations is also performed, in which mortar joints are reduced to interfaces and blocks are supposed infinitely resistant.

The standard deviation for blocks length is assumed equal to $60 \mathrm{~mm}$ whereas blocks height is assumed constantly equal to $120 \mathrm{~mm}$. Wall thickness is assumed equal to $100 \mathrm{~mm}$.

A typical heterogeneous discretization by means of triangular elements with interfaces discontinuities for the wall under consideration is depicted in Figure 19-b, whereas the mesh used when dealing with the homogenization approach is reported in Figure 19-c.

The aim of the example is to compare failure load distributions, namely mean value, standard deviation and possible non Gaussian behavior, and failure mechanisms provided by the homogenized and the heterogeneous model. In this structure the collapse is due to the formation of a central plastic bending hinge, and hence tensile masonry strength along the horizontal axis is predominant. Since horizontal masonry strength is somewhat dependent on blocks staggering (see for instance [20]), the output value of the structural limit analysis (failure load) has a statistical distribution strictly connected to blocks length variability.

It is worth noting that, as a rule, a single simulation on the heterogeneous model required around 20 minutes to be performed, whereas less than 120 seconds were required for the homogenized approach. This justifies the relatively limited number of simulations performed $(2,000)$ to evaluate the output distributions, which in any case required around 30 days of processing time only for the heterogeneous model. Considering also that three hours were required to collect the database of failure surfaces (see previous sections) for the homogenized approach, around one day was needed to complete the homogenized simulations, meaning that the simple two steps limit analysis procedure here proposed competes very favorably with direct models based on a distinct discretization of blocks and joints.

In Figure 21, a comparison between deformed shapes at collapse obtained with a typical heterogeneous mesh and the homogenized approach (final mesh after 14 adaptations) is represented (failure mechanisms corresponding to the average collapse load value). As it is possible to notice, an asymmetric failure is reached, which is obviously a consequence of the asymmetric disposition of the blocks. In any case, the failure mechanism provided by the heterogeneous model is almost perfectly reproduced by the homogenized approach. Finally, in Figure 22, the failure load 
distribution provided by the heterogeneous and the homogenized approach are compared, along with the corresponding confidence bounds. The distributions are quite similar, with rather similar average values (5\% difference) and standard deviations (1\% difference). As expected, the behavior of both distributions is almost Gaussian (kurtosis value of three), with very low asymmetry (skewness of zero).

\section{Masonry shear wall}

A masonry shear wall, originally tested with a regular disposition of bricks by Raijmakers and Vermeltfoort [31], is here re-analyzed with a random disposition of bricks. The width/height ratio $(\mathrm{L} / \mathrm{H})$ of the shear walls is $990 / 1000([\mathrm{~mm}] /[\mathrm{mm}])$; the walls were built up with 18 courses of bricks, from which 16 courses were active and 2 were clamped in stiff steel beams, Figure 23 . The brick dimensions are 210 (mean value) $52 \times 100 \mathrm{~mm}^{3}$ and the mortar joints are $10 \mathrm{~mm}$ thick. A vertical pre-compression load $p$ equal to $2.12 \mathrm{~N} / \mathrm{mm}^{2}$ is applied on the top, keeping the resultant vertical load constant during the complete loading procedure. The stiff steel beam did not allow rotations of the top and was subsequently pushed with an increasing horizontal force.

In order to preclude top horizontal beam rotations, in the limit analysis simulations, vertical displacements of the nodes belonging to the horizontal top edge were constrained to be all equal.

Analogously to the previous case, two random bricks assemblages are analyzed, corresponding respectively to the arrangements depicted in Figure $2-\mathrm{a}$ and $-\mathrm{b}$ and extensively analyzed at a cell level in the previous section.

In both models, for mortar joints a Lourenço and Rots [1] failure criterion is used, with mechanical properties summarized in Table III.

\subsection{No horizontal alignment (Case I disposition): homogenized analyses}

As for the deep beam example, a preliminary set of Monte Carlo simulations is performed on the shear wall, discussing the effect of changing the COV of height and length blocks and assuming a disposition of blocks without horizontal alignment (Case I arrangement). Six different values of $\mathrm{COV}$ for $\mathrm{H}$ and $\mathrm{L}$ are investigated, respectively equal to $2.5-5-10-15-20-25 \%$. At fixed values of $\mathrm{H}$ and L COVs, 2,000 structural analyses are repeated in order to obtain empirical cumulative distribution functions (ECDFs) of the failure load from the homogenized model. In Figure 24, numerical simulations results are depicted, representing ECDFs of the failure total shear at the base corresponding to fixed length and height COVs. Estimated distributions mean values, deterministic 
failure loads assuming a running bond and a stack bond disposition are also represented, using respectively black, red and purple thick curves.

Similarly to what pointed out for the deep beam case, when a COV near to zero for both the height and the length of the blocks (upper left diagram of Figure 24) is considered, the wall approximates a stack bond disposition. Intuitively, failure load standard deviation tends to increase increasing block dimensions COVs. Analogously to the previous case, the upper bound is not theoretically represented by a running bond texture, because an extra resistance (both in shear and in vertical membrane action) may be present in Case I, due to full staggering of the blocks. Moreover, the stack bond lower bound is well approximated by the low COVs, which is again due to the adopted definition of geometry, and not necessarily represents the physics of irregular masonry bonds.

Finally, it is worth noting that, considering that both failure is dominated by shear resistance and standard deviation of strength distributions in pure shear is smaller than the horizontal one (compare, for instance Figure 10-b and Figure 12-c), it is very straightforward to conclude that failure load standard deviation is generally smaller compared to that of the deep beam example.

\subsection{Case II disposition: quasi periodic arrangement}

A second sub-set of Monte Carlo simulations is performed for the case at hand, assuming a quasiperiodic blocks disposition (Case II of Figure 2), with a standard deviation for bricks length equal to $50 \mathrm{~mm}$.

Meshes used for the heterogeneous and the homogenized limit analyses are depicted in Figure 23. Two rows of elements at the base and at the top of the wall (blue or darker triangles in Figure 23) have been assumed infinitely resistant in order to model the effect of the stiff steel beam. Homogenized mesh of Figure 23 refers to the mesh used at the beginning of the SLP mesh adaptation.

The aim of the example is to compare failure loads and failure mechanisms provided by the homogenized and the heterogeneous model in a case where the collapse is due to the formation of a inclined strut, with a plastic region of significant dimension at the compressed toe. Differently to the previous example, a complex interaction among tensile, compressive and shear strength of masonry contribute to the overall resistance of the wall.

Due to the relatively small number of elements required for a heterogeneous discretization, only two minutes and less than 20 seconds were required for a single heterogeneous and homogenized Monte Carlo simulation respectively. Hence, a total of 10,000 simulations to evaluate the output collapse load distribution have been performed, requiring around 20 days of processing time for the heterogeneous approach. Also in this case, the homogenization model competes favorably with 
micro-modeling, requiring only three days, comprising 10,000 simulations and the preliminary evaluation of the stochastic failure surface.

In Figure 25, a comparison between deformed shapes at collapse obtained with a heterogeneous mesh and the homogenized one (final mesh after eight adaptations) is reported (failure mechanisms correspond to the average collapse load values). As it is possible to notice, an almost perfect agreement between deformed shapes is obtained, meaning that the procedure proposed may be used for a reliable evaluation of failure mechanisms and collapse loads.

Finally, in Figure 26, failure loads distributions provided by the heterogeneous and the homogenized approach are represented, with the corresponding confidence bounds. Again, the distribution provided by the homogenized approach is very similar to the heterogeneous one, with comparable average values (2\% difference) and standard deviations (6\% difference). As expected, the behavior of both distributions is almost Gaussian, with low asymmetry.

\section{Conclusions}

A simple homogenized rigid-plastic plate model for a fast and reliable analysis of masonry structures constituted by blocks disposed in irregular texture has been presented. A two-steps approach has been adopted, including a preliminary homogenization of the random assemblage of bricks, followed by structural Monte Carlo homogenized FE analyses to compare to full expensive stochastic heterogeneous approaches.

A full description of the model has been given considering a representative volume constituted by a generic regular block with parallelepiped shape interconnected with its neighbors. A sub-class of possible elementary deformation modes (mimicking horizontal and vertical stretching and a pure shear deformation) acting in the unit cell has been a priori chosen in order to describe joints cracking under normal and tangential actions. Then, power dissipated in the discrete model has been equated to that dissipated in a continuum macroscopic 2D equivalent plate. Such identification is based on a simple correspondence between motions in the 3D discrete model and the continuum. Following what was presented in the case of regular assemblages of blocks, joints have been reduced to interfaces with a cohesive associated frictional behavior with limited tensile and compressive strength, whereas blocks have been supposed infinitely resistant.

Two different dispositions have been investigated in detail, assuming that block dimensions exhibit a stochastic variability with predetermined random distribution. In this way, the horizontal position of vertical joints as well as the vertical position of horizontal joints has been treated as statistically variable. 
After a fast numerical evaluation of stochastic masonry failure surfaces to use (step I), a masonry deep beam and a shear wall have been analyzed at a structural. In the first case, failure is mostly controlled by the tensile strength parallel to the joints, link to the staggering of the joints, whereas, in the second case, a complex failure mode involving tension, shear and crushing is present. The speed up of the proposed methodology in the case studies presented is in the order of 10 to 30 .

\section{References}

[1] Lourenço PB, Rots J (1997). A multi-surface interface model for the analysis of masonry structures. Journal of Engineering Mechanics ASCE 123 (7): 660-668.

[2] Milani G (2008). 3D upper bound limit analysis of multi-leaf masonry walls. International Journal of Mechanical Sciences 50: 817-836.

[3] Milani G, Zuccarello FA, Olivito RS, Tralli A (2007). Heterogeneous upper-bound finite element limit analysis of masonry walls out-of-plane loaded. Computational Mechanics 40 (6): 911-931.

[4] Lotfi HR, Shing PB (1994). Interface model applied to fracture of masonry structures. Journal of Structural Engineering ASCE 120(1): 63-80.

[5] Lourenço PB, de Borst R, Rots J (1997). A plane stress softening plasticity model for orthotropic materials. International Journal for Numerical Methods in Engineering 40: 40334057.

[6] Casolo S (1999). Rigid Element Model for Non-Linear Analysis of Masonry Facades Subjected to out-of-Plane Loading. Communications in Numerical Methods in Engineering 15(7): 457-468.

[7] Pegon P, Anthoine A (1997). Numerical strategies for solving continuum damage problems with softening: application to the homogenization of masonry. Computers and Structures 64(4): 623-642.

[8] Luciano R, Sacco E (1997). Homogenization technique and damage model for old masonry material. International Journal of Solids and Structures 34(4): 3191-208.

[9] Pietruszczak S, Ushaksaraei R (2003). Description of inelastic behaviour of structural masonry. International Journal of Solids and Structures 40: 4003-4019.

[10] Massart T, Peerlings RHJ, Geers MGD (2004). Mesoscopic modeling of failure and damageinduced anisotropy in brick masonry. European Journal of Mechanics A/Solids 23: 719-735. 
[11] Mercatoris BCN, Massart TJ (2009). Assessment of periodic homogenisation-based multiscale computational schemes for quasi-brittle structural failure. International Journal for Multiscale Computational Engineering 7(2): 153-170.

[12] Cluni F, Gusella V (2004). Homogenization of non-periodic masonry structures. International Journal of Solids and Structures 41: 1911-1923.

[13] Falsone G, Lombardo M (2007). Stochastic representation of the mechanical properties of irregular masonry structures. International Journal of Solids and Structures 44: 8600-8612.

[14] Cecchi A, Sab K (2009). Discrete and continuous models for in plane loaded random elastic brickwork. European Journal of Mechanics A-Solids 28: 610-625.

[15] Gusella V, Cluni F (2006). Random field and homogenization for masonry with nonperiodic microstructure. Journal of Mechanics of Materials and Structures 1(2): 357-386.

[16] Cecchi A, Sab K (2009). A homogenized Love-Kirchhoff model for out-of-plane loaded Random 2D lattices. Application to Brickwork Panels. International Journal of Solids and Structures 46(14-15): 2907-2919.

[17] de Buhan P, de Felice G (1997). A homogenization approach to the ultimate strength of brick masonry. Journal of the Mechanics and Physics of Solids 45 (7): 1085-1104.

[18] Ferris M, Tin-Loi F (2001). Limit analysis of frictional block assemblies as a mathematical program with complementarity constraints. Int. J. Mech. Sci. 43: 209-224.

[19] Orduña A, Lourenço PB (2005). Three-dimensional limit analysis of rigid blocks assemblages. Part I: Torsion failure on frictional joints and limit analysis formulation. Int. J. Solids and Structures 42 (18-19): 5140-5160.

[20] Milani G, Lourenço PB, Tralli A (2006). Homogenised limit analysis of masonry walls. Part I: failure surfaces. Computers and Structures 84 (3-4): 181-195.

[21] Milani E, Milani G, Tralli A (2008). Limit analysis of masonry vaults by means of curved shell finite elements and homogenization. International Journal of Solids and Structures 45: 5258-5288.

[22] Cecchi A, Milani G (2008). A kinematic FE limit analysis model for thick English bond masonry walls. International Journal of Solids and Structures 45(5): 1302-1331.

[23] Milani G (2009). Homogenized limit analysis of FRP-reinforced masonry walls out-of-plane loaded. Computational Mechanics 43: 617-639. 
[24] Begg D, Fishwick R (1995). Numerical analysis of rigid block structures including sliding. In: Middleton J, Pande G (Eds.), Computer Methods in Structural Masonry 3: 177-183.

[25] Suquet P (1983). Analyse limite et homogeneisation. Comptes Rendus de l'Academie des Sciences - Series IIB - Mechanics 296: 1355-1358.

[26] Sab K (2003). Yield design of thin periodic plates by a 25omogenization technique and an application to masonry walls. C.R. Mechanique 331: 641-646.

[27] Page AW (1981). A biaxial failure criterion for brick masonry in the tension-tension range. International Journal of Masonry 1: 26-30.

[28] Milani G, Lourenço PB (2009). A discontinuous quasi-upper bound limit analysis approach with sequential linear programming mesh adaptation. International Journal of Mechanical Sciences 51(1): 89-104.

[29] Sloan SW, Kleeman PW (1995). Upper bound limit analysis using discontinuous velocity fields. Computer Methods in Applied Mechanics and Engineering 127 (1-4): 293-314.

[30] Krabbenhoft K, Lyamin AV, Hjiaj M, Sloan SW (2005). A new discontinuous upper bound limit analysis formulation. International Journal for Numerical Methods in Engineering 63: 1069-1088.

[31] Raijmakers TMJ, Vermeltfoort A (1992). Deformation controlled tests in masonry shear walls (in Dutch). Report B-92-1156, TNO-Bouw, Delft, The Netherlands. 


\section{Figures}

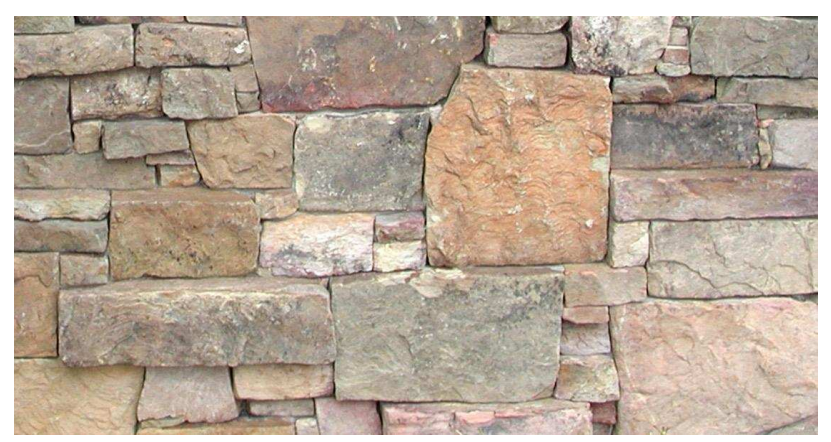

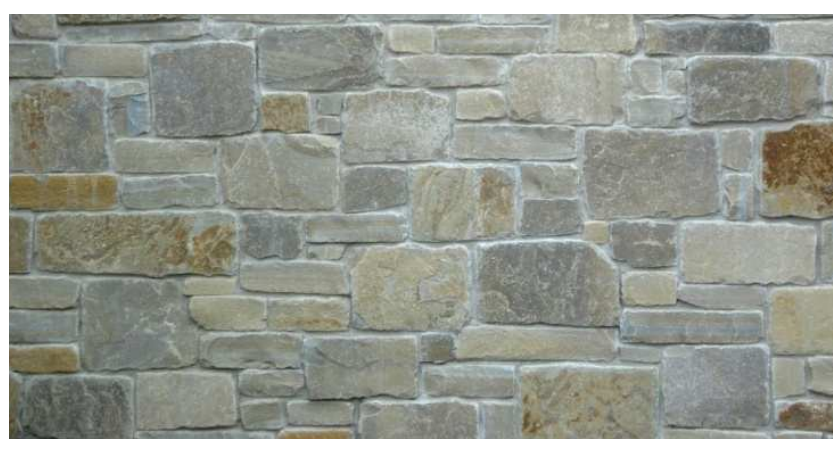

$-a$

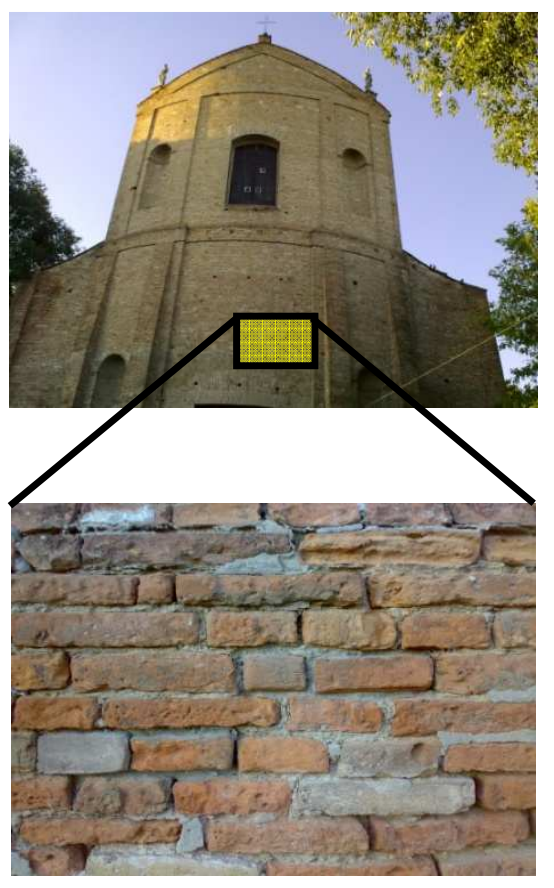

$-b$

Figure 1: Typical random patterns in existing historical buildings. -a: without horizontal alignment. -b: with horizontal alignment.
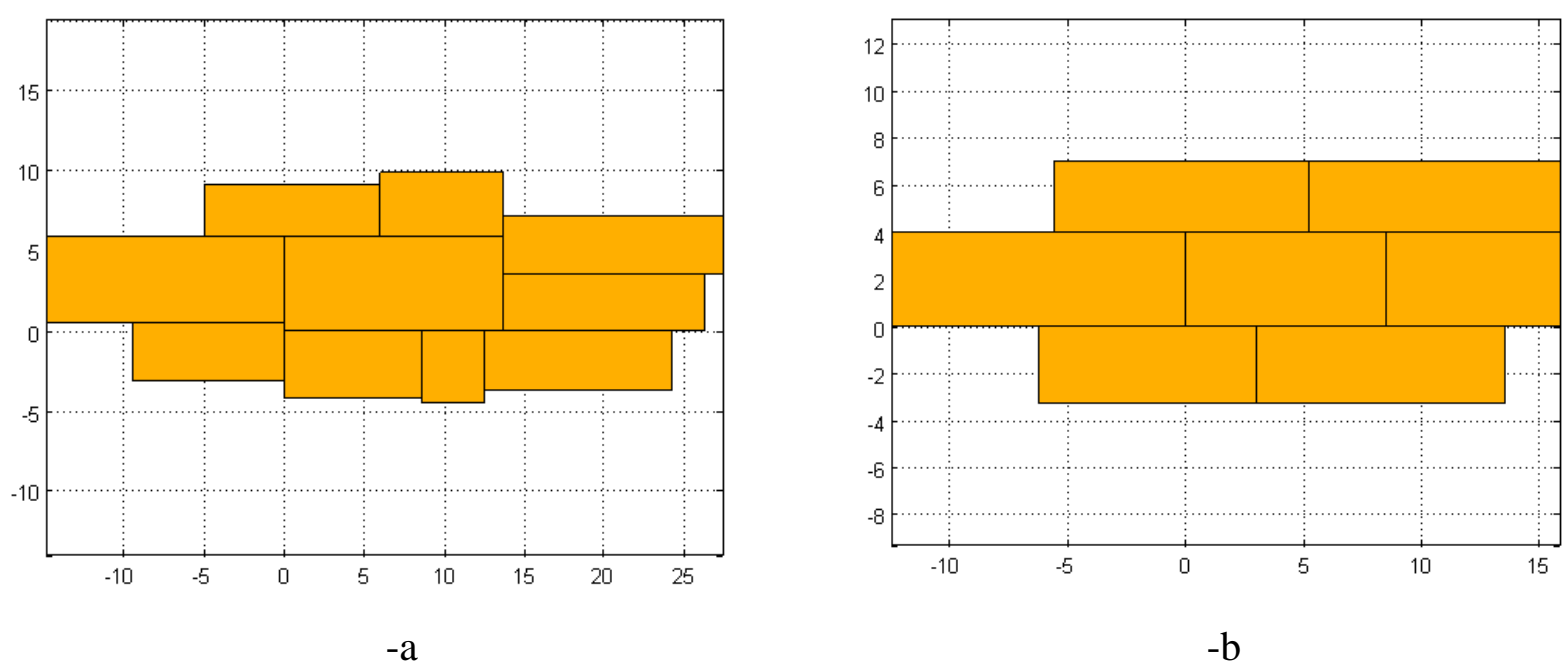

Figure 2: Randomly generated REVs. -a: without horizontal alignment (Case I). -b: with horizontal alignment (Case II). 


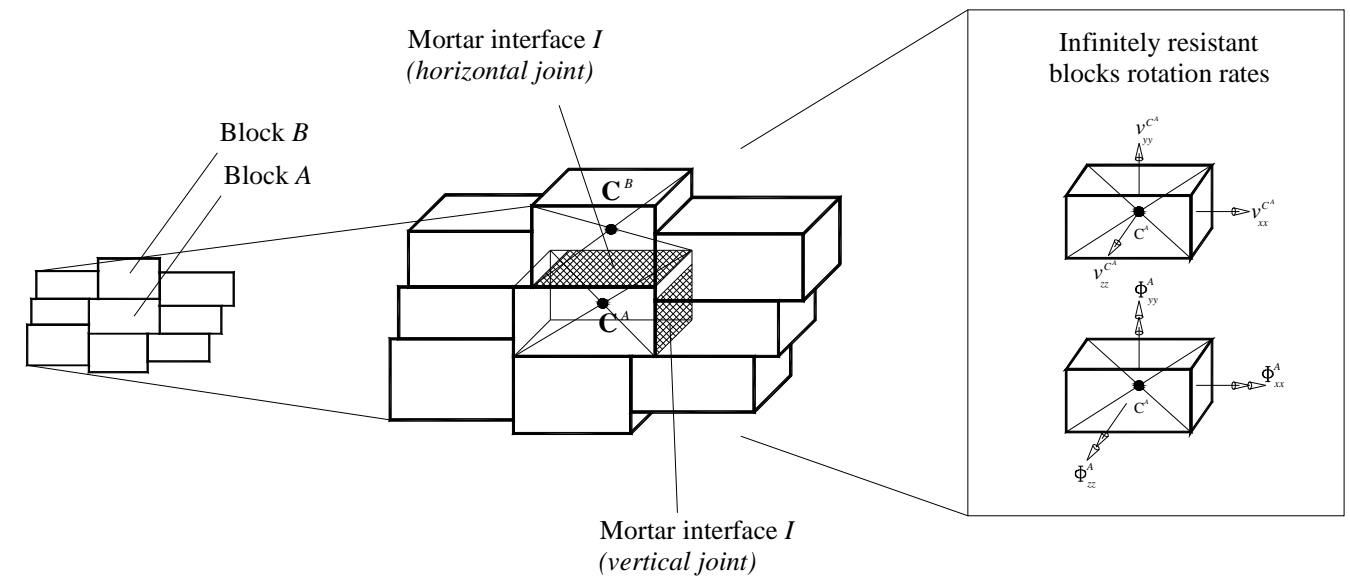

Figure 3: Masonry kinematic model. Two adjacent blocks ( $A$, centroid $\mathbf{C}^{A}$ and $B$, centroid $\mathbf{C}^{B}$ ) connected by means of a mortar interface $I$ where plastic dissipation occurs. For each block three velocities unknowns and three rotation rates must be introduced in the optimization problem at a cell level (infinite strength of blocks hypothesis).

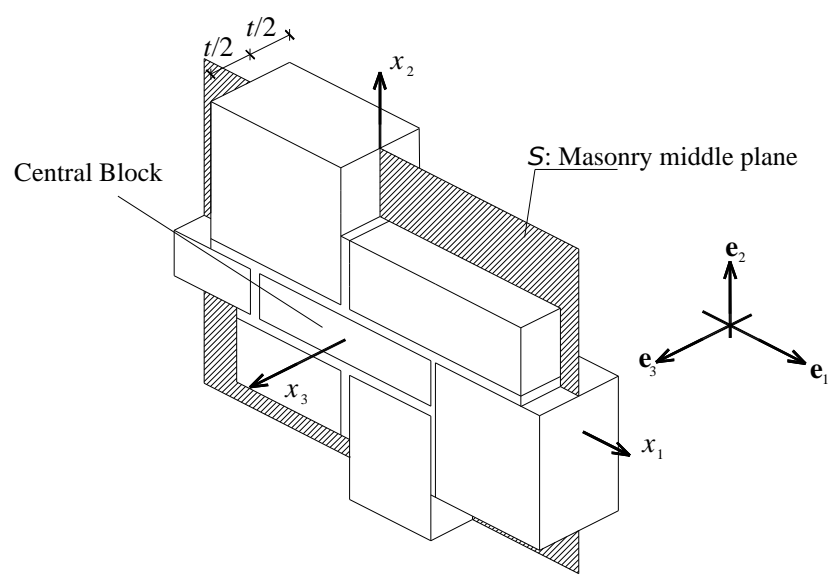

Figure 4: Reference surface chosen for masonry. 


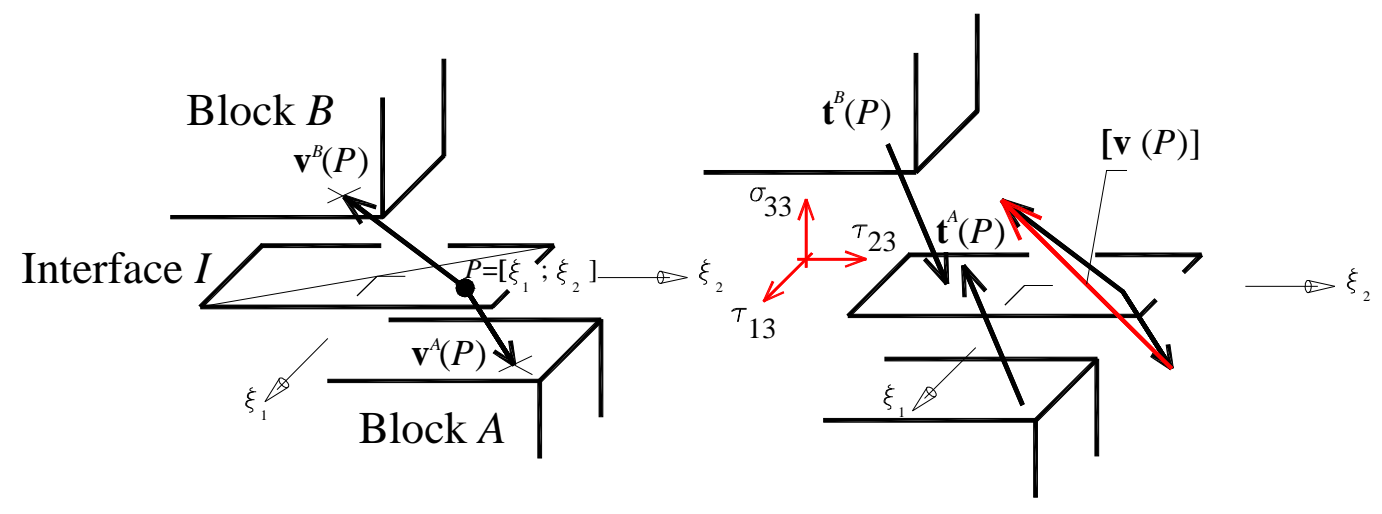

Figure 5: Jump of velocities and stress field acting on an interface $I$ between contiguous blocks $A$ and $B$. 


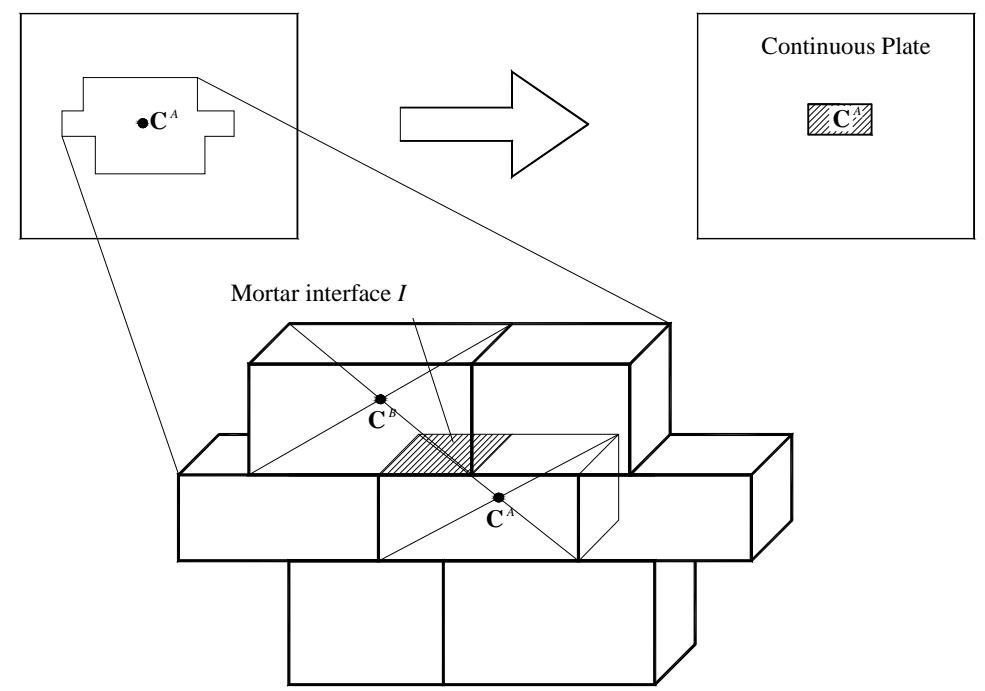

Figure 6: Representative volume element and identification between discrete model and continuous model. 


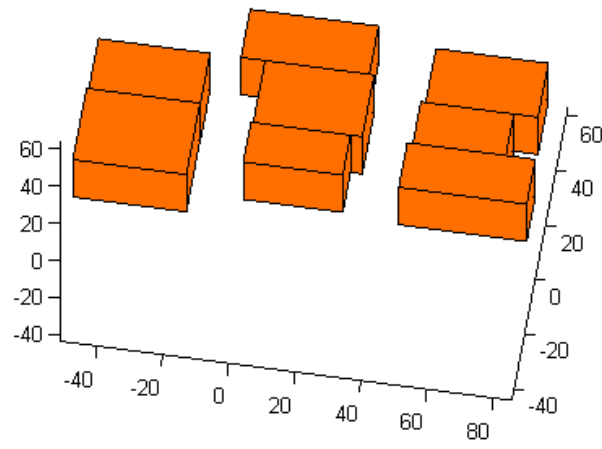

$-a$

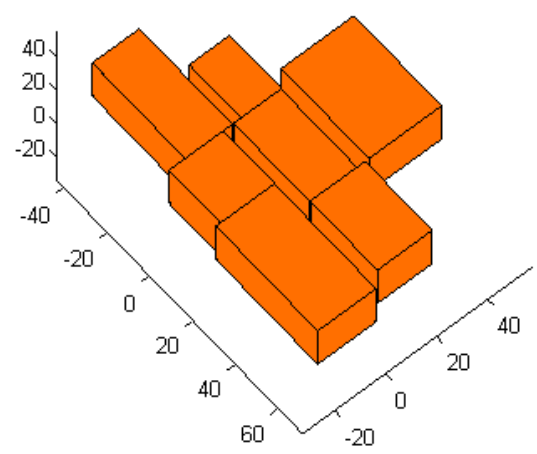

$-\mathrm{C}$

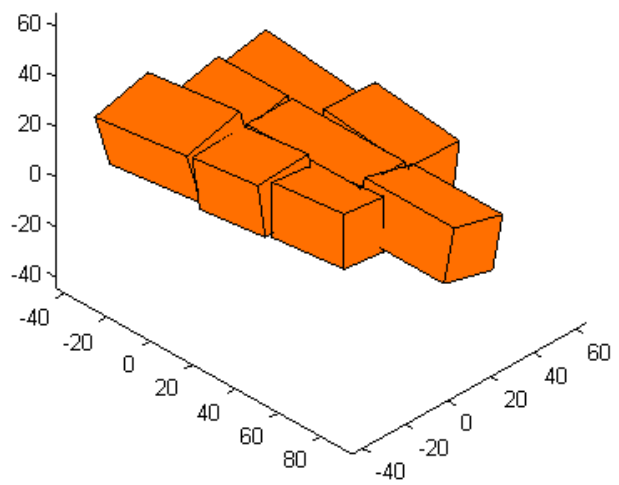

$-e$

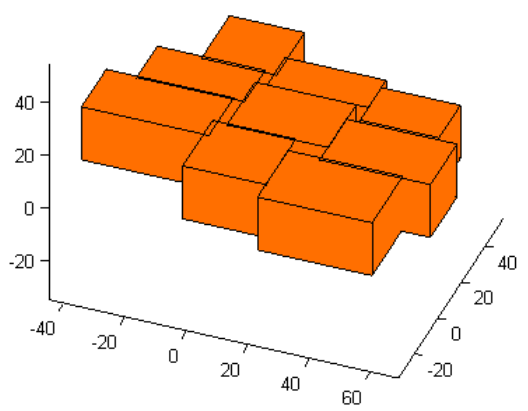

$-b$

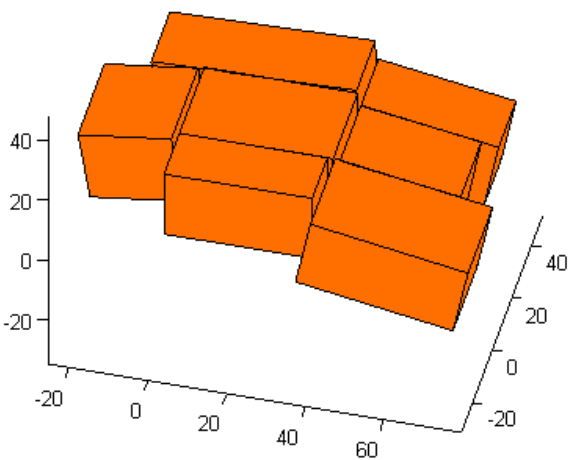

$-d$

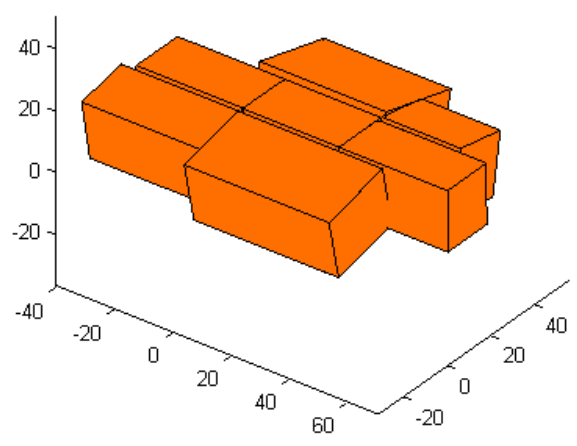

$-\mathrm{f}$

Figure 7: Elementary homogeneous deformations applied to the representative volume element. - a: $\dot{E}_{11},-$ b: $\dot{E}_{12},-$ c: $\dot{E}_{22},-$ d: $\dot{\chi}_{11},-$ e: $\dot{\chi}_{12},-$ f: $\dot{\chi}_{22}$. 

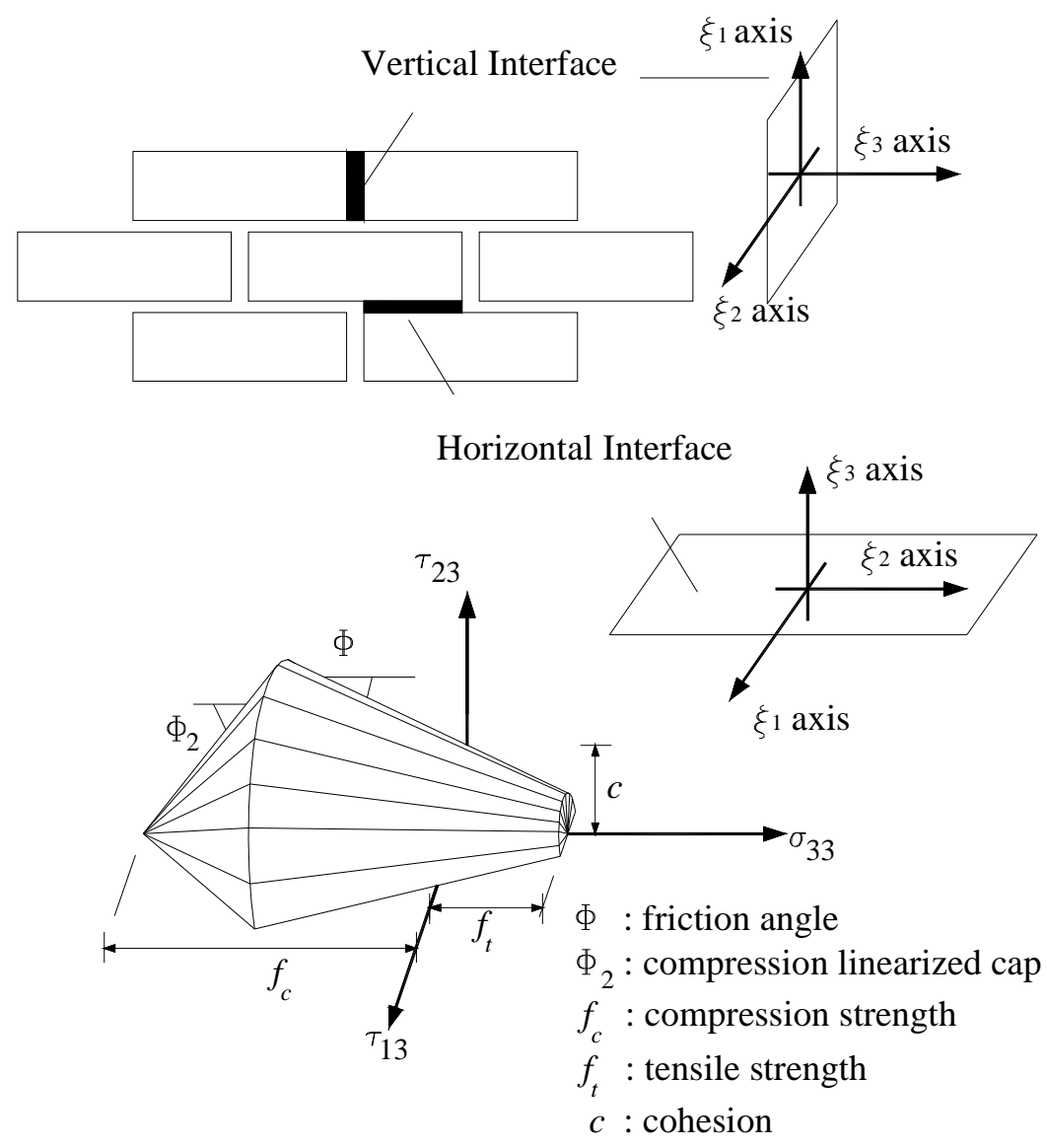

Figure 8: Piecewise linear approximation of the failure criterion adopted for joints. MohrCoulomb failure criterion with tension cut-off and linearized compression cap. 

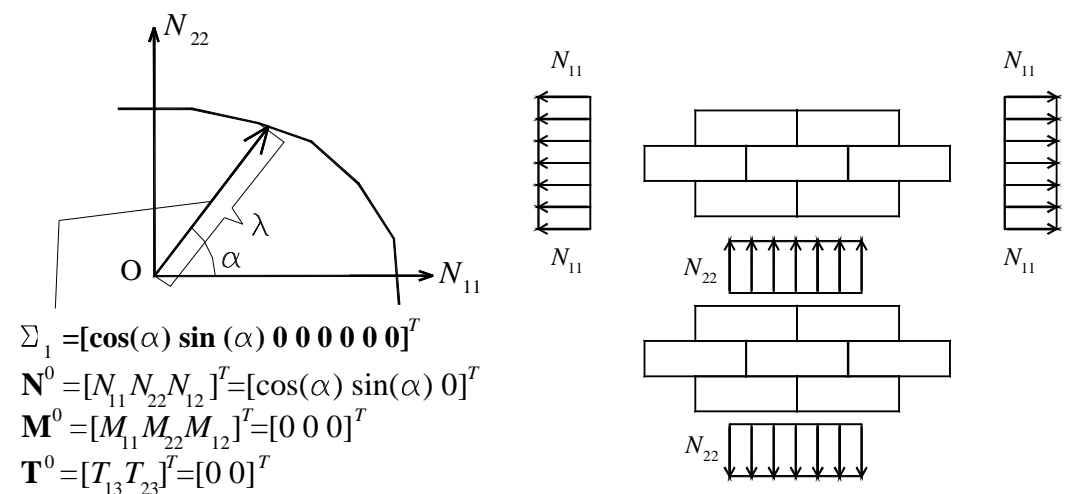

Figure 9: Meaning of $\lambda$ multiplier in the optimization problem and $\alpha$ angle. 


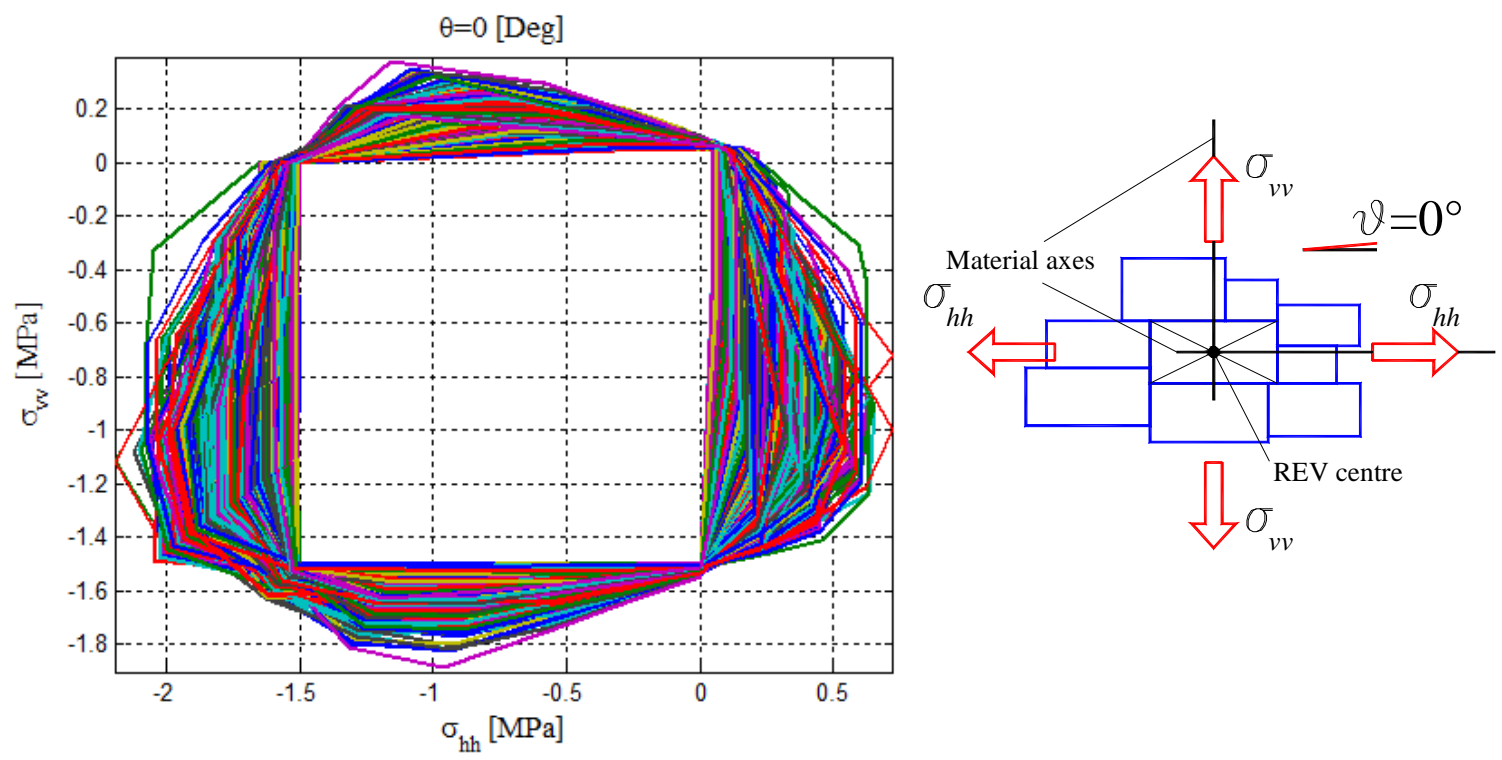

Figure 10: Case I results, $\vartheta=0^{\circ}$. Monte Carlo failure surfaces in the plane $\sigma_{h h}-\sigma_{v v}$. 


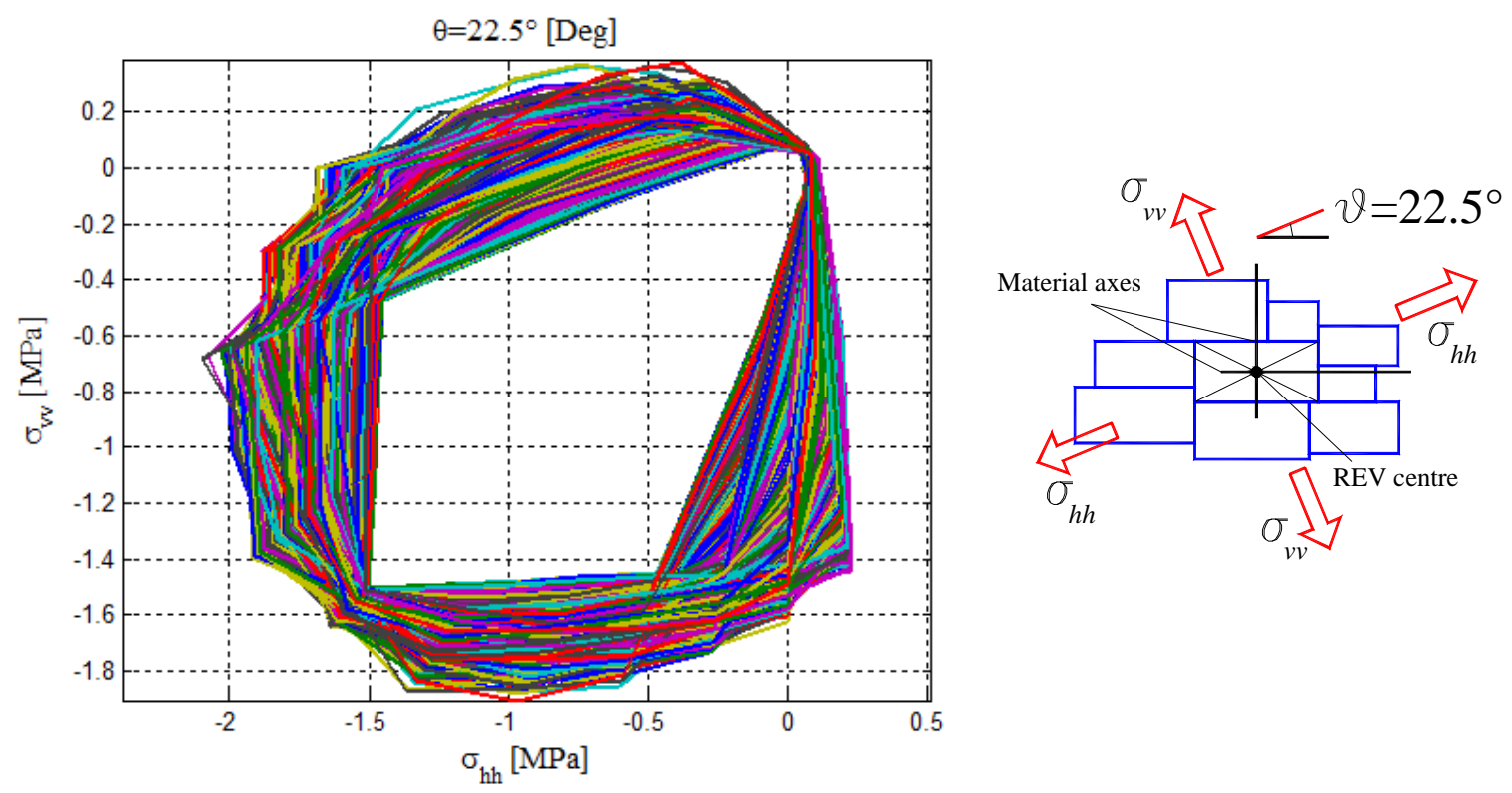

Figure 11: Case I results, $\vartheta=22.5^{\circ}$. Monte Carlo failure surfaces in the plane $\sigma_{h h}-\sigma_{v v}$. 


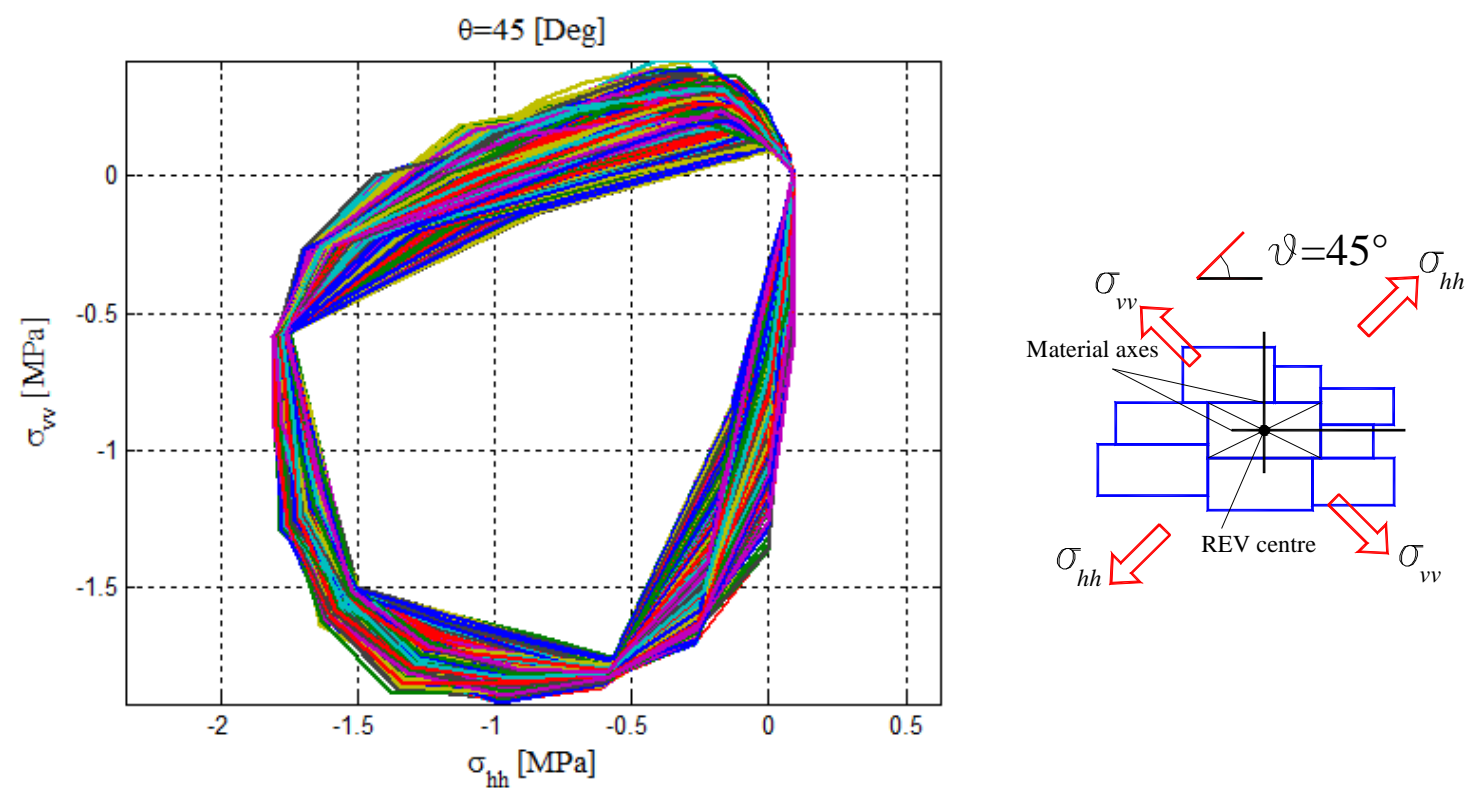

Figure 12: Case I results, $\vartheta=45^{\circ}$. Monte Carlo failure surfaces in the plane $\sigma_{h h}-\sigma_{v v}$. 

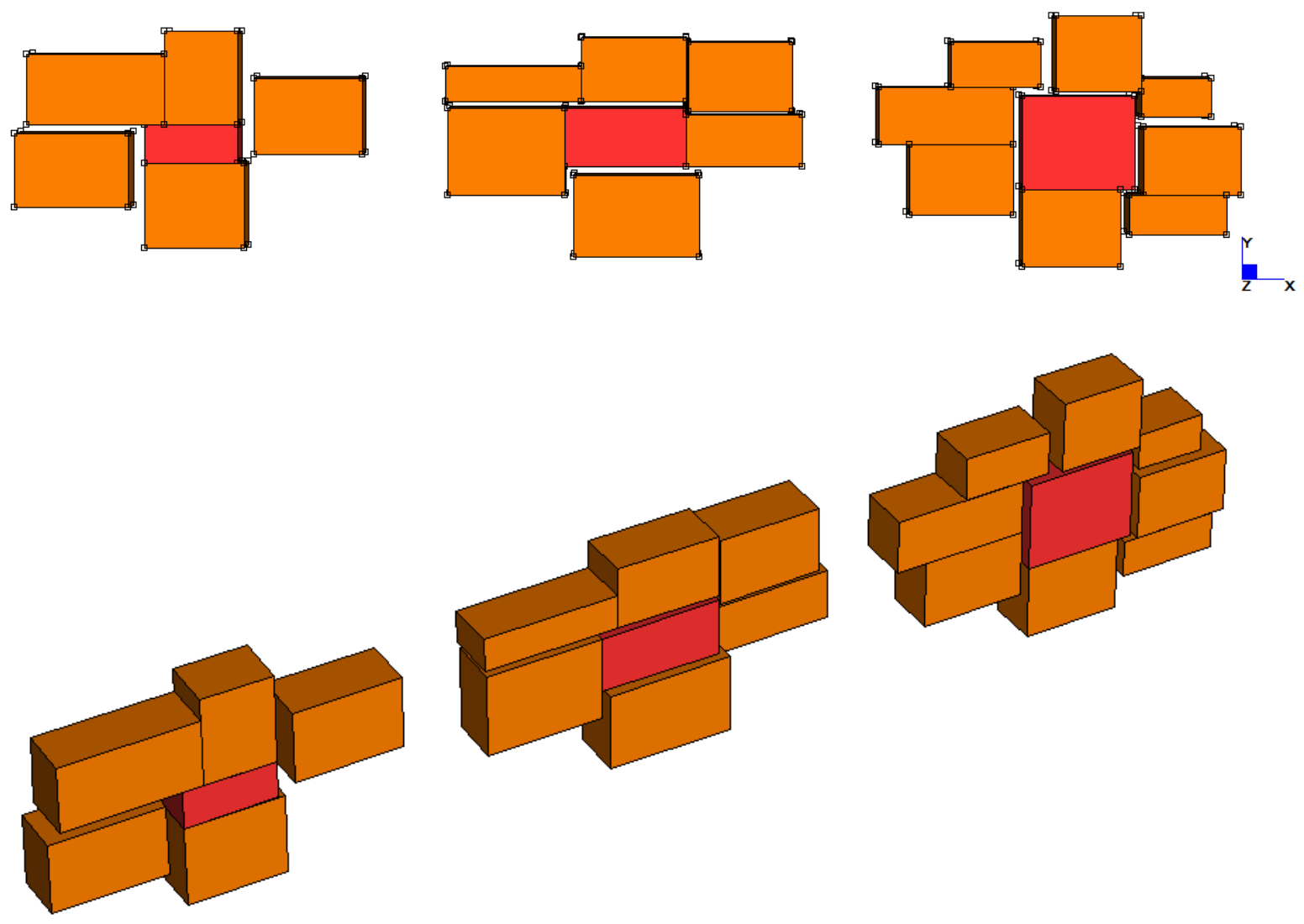

Figure 13: Case I. Some typical deformed shape at collapse obtained with the kinematic model proposed. 


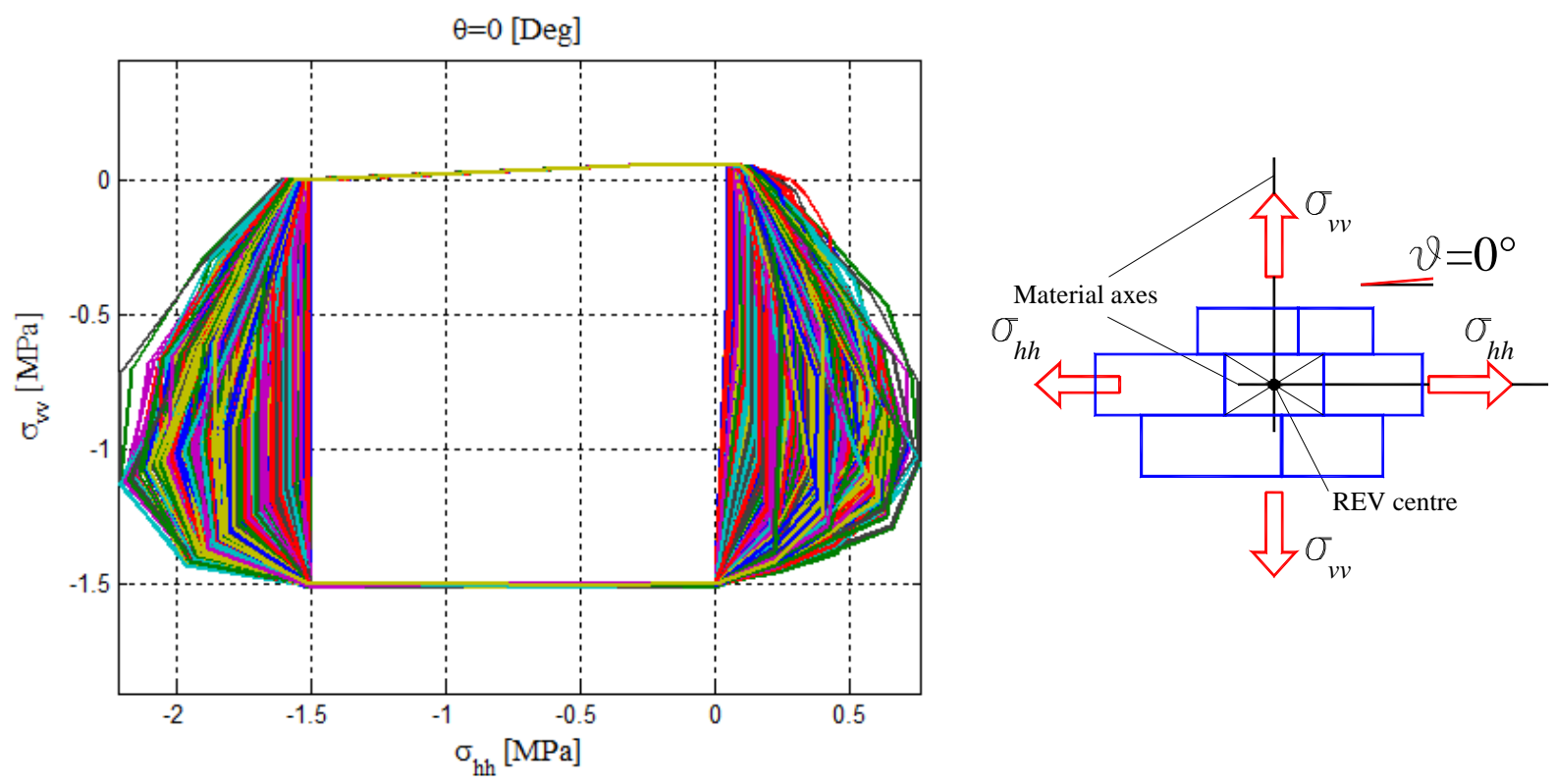

Figure 14: Case II results, $\vartheta=0^{\circ}$. Monte Carlo failure surfaces in the plane $\sigma_{h h}-\sigma_{v v}$. 


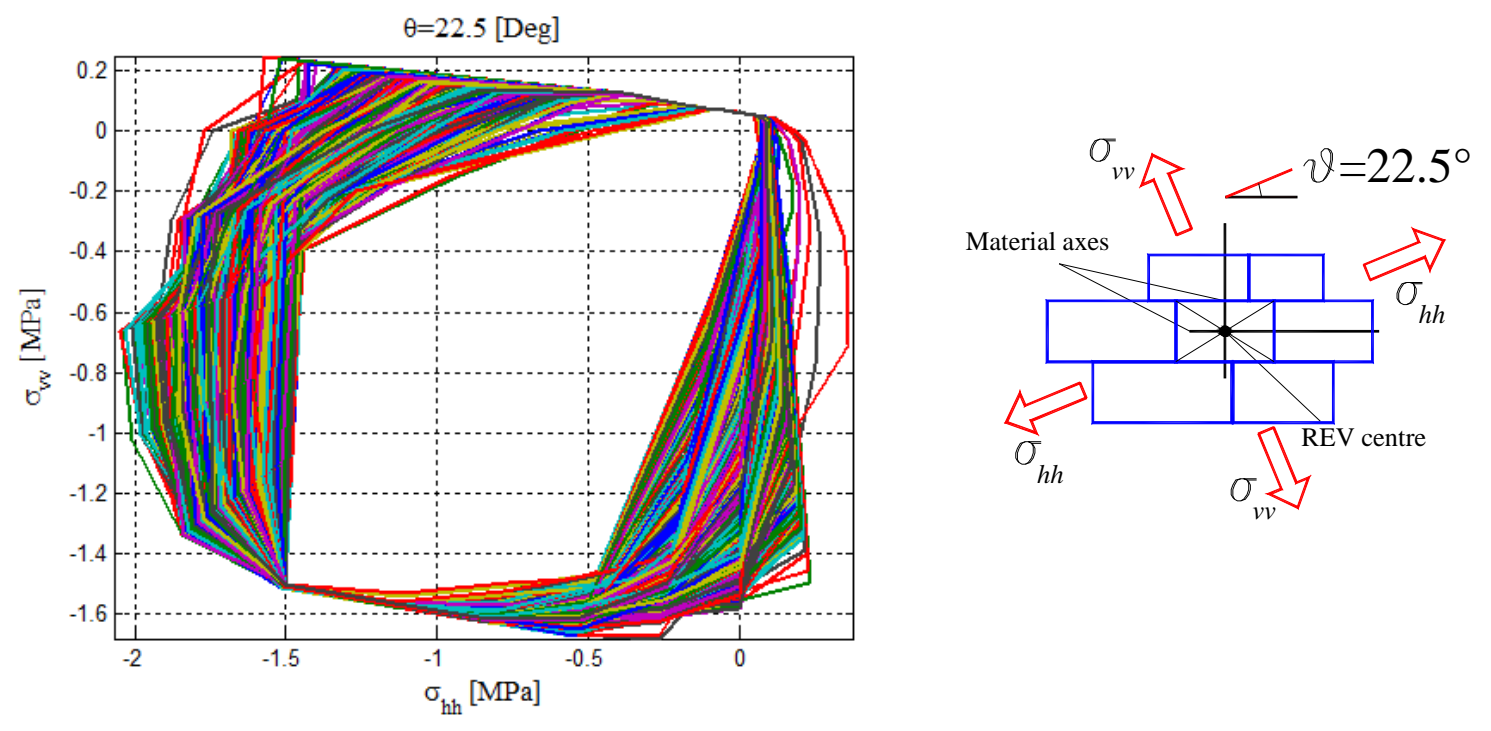

Figure 15: Case II results, $\vartheta=22.5^{\circ}$. Monte Carlo failure surfaces in the plane $\sigma_{h h}-\sigma_{v v}$. 


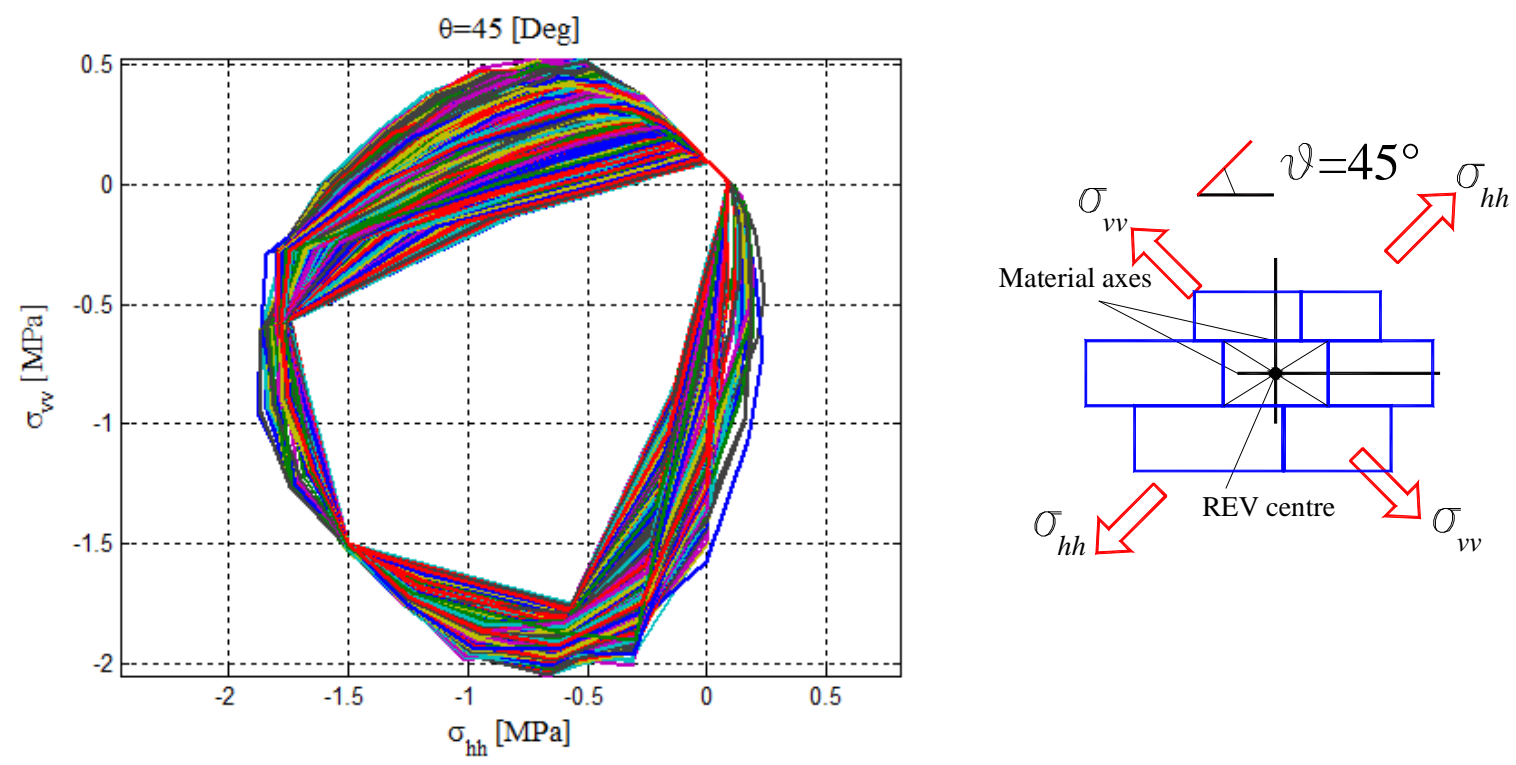

Figure 16: Case II results, $\vartheta=45^{\circ}$. Monte Carlo failure surfaces in the plane $\sigma_{h h}-\sigma_{v v}$ 

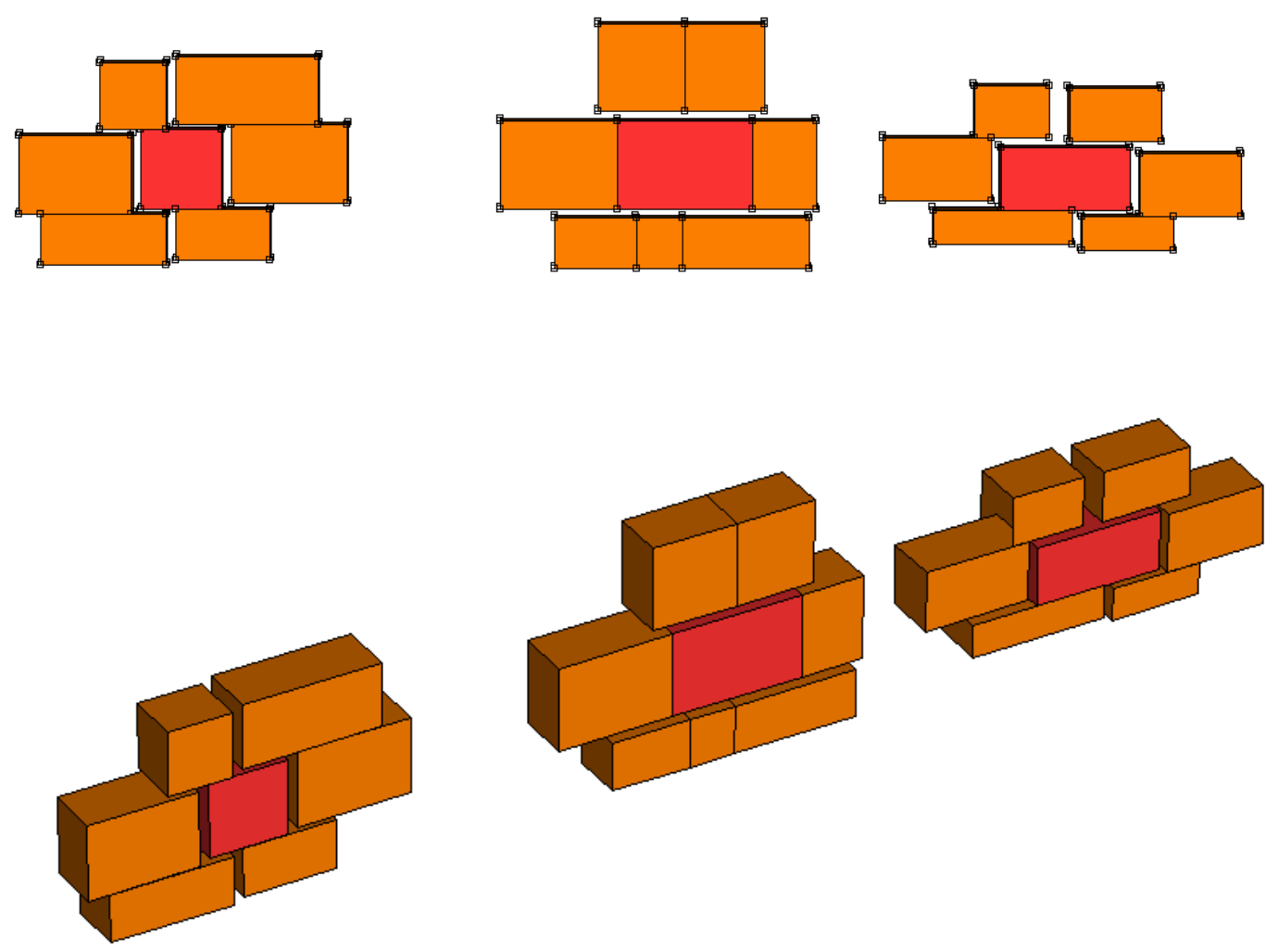

Figure 17: Case II. Some typical deformed shape at collapse obtained with the kinematic model proposed.
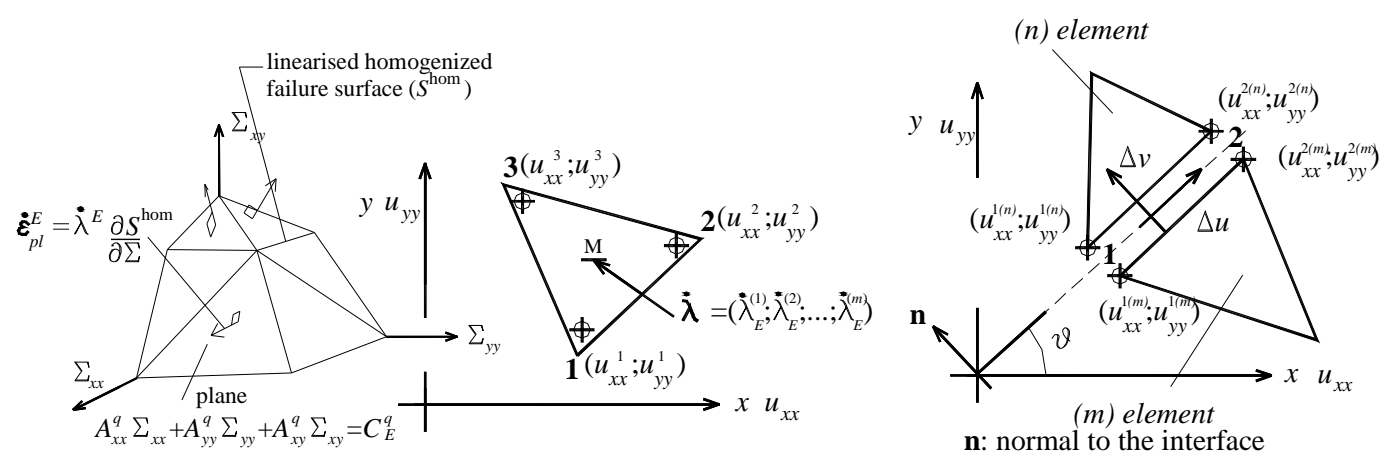

$-\mathrm{a}$

$-b$

$-\mathrm{c}$

Figure 18: Finite element used for the structural stochastic limit analysis. 

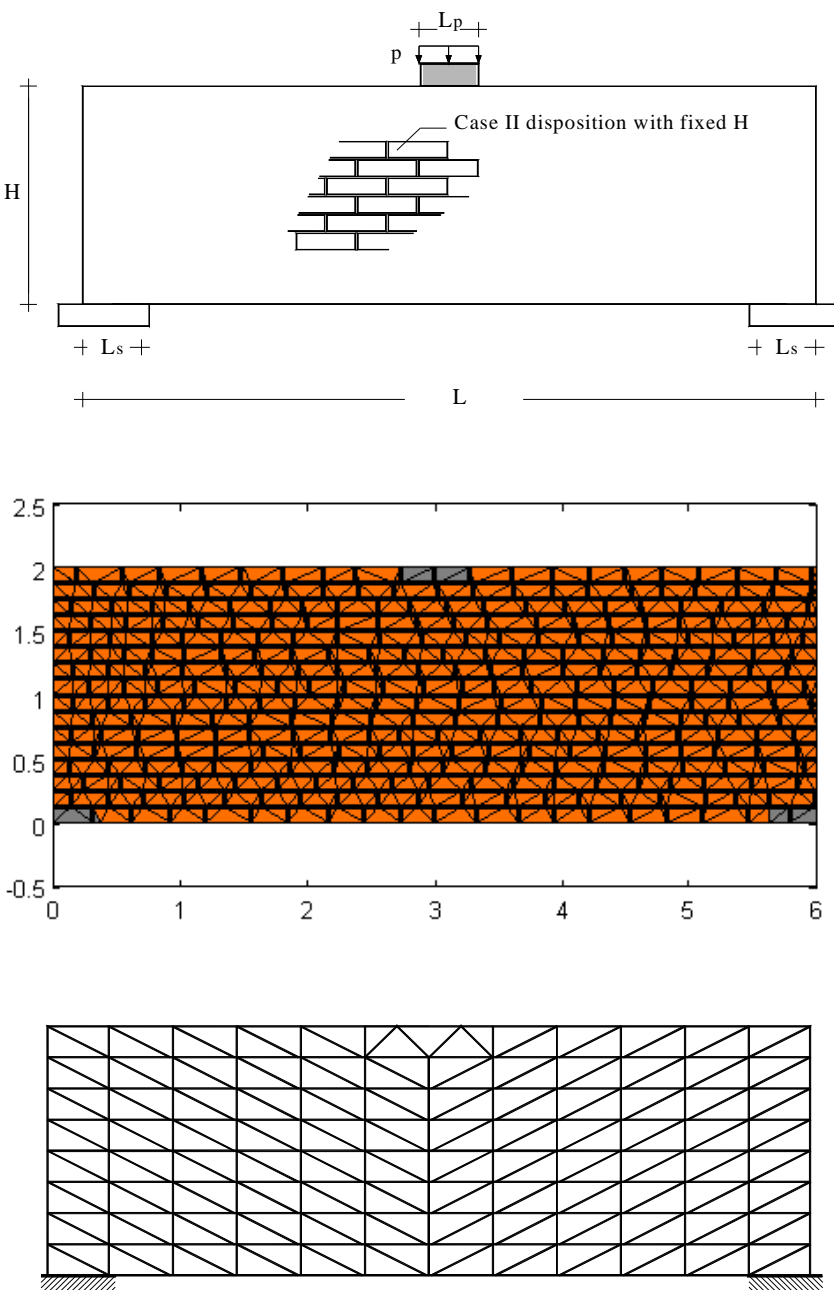

$-\mathrm{c}$

Figure 19: Deep beam example. Geometry, loading and constraints condition (-a), typical heterogeneous (-b) and homogenized mesh (-c) used. 

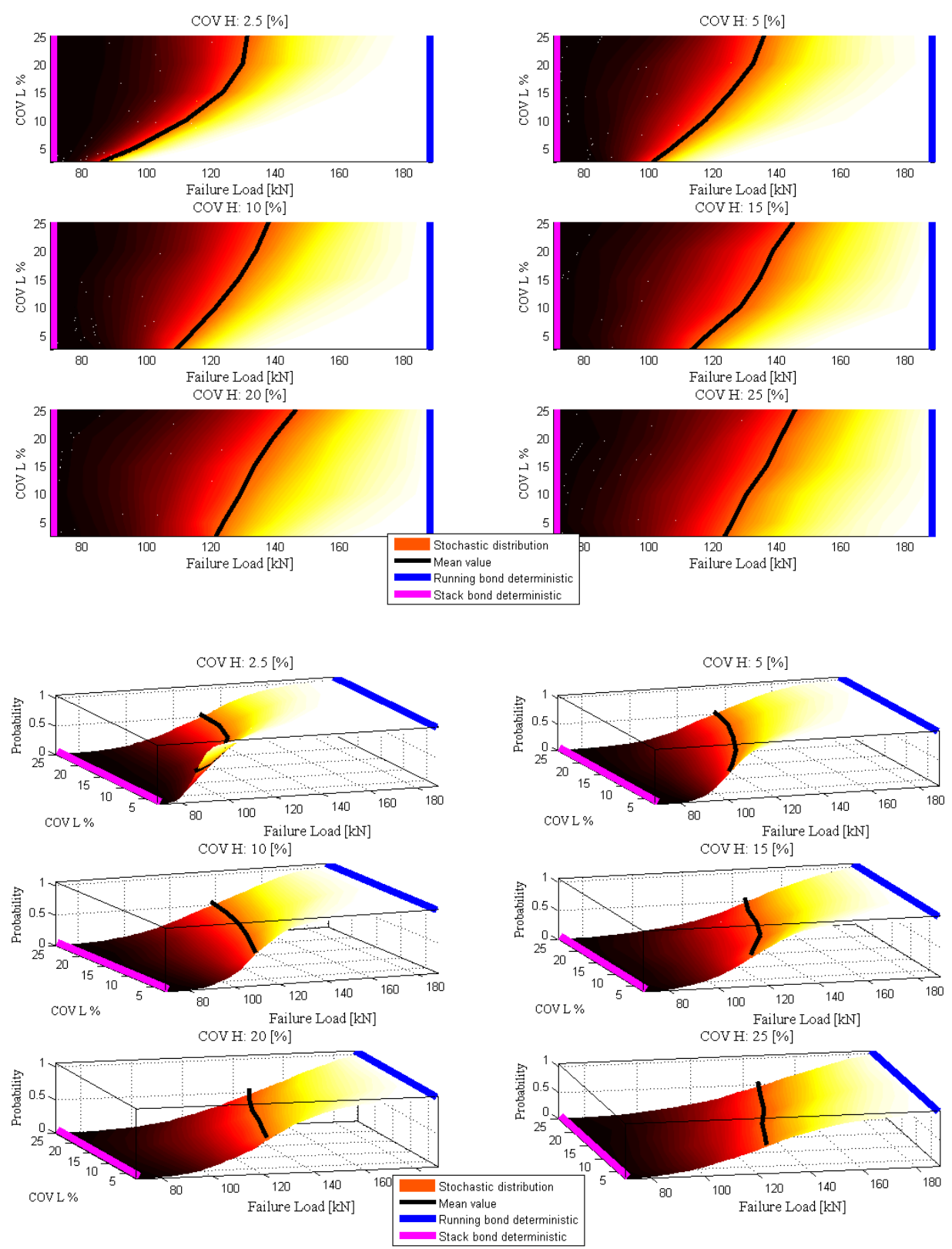

Figure 20: Deep beam example. Failure load distribution at different COVs of blocks length and height (Case I). 

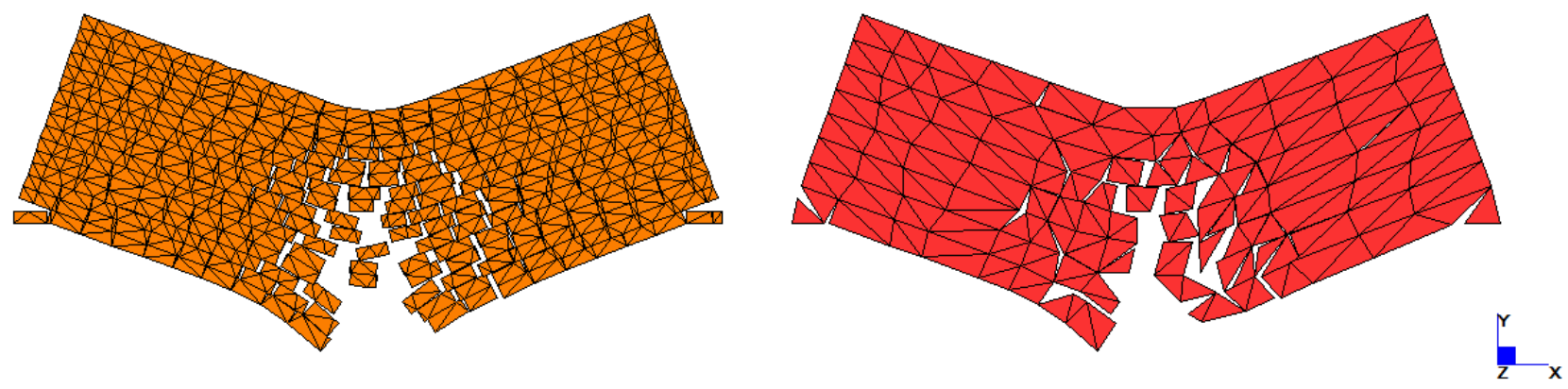

Figure 21: Deep beam example. Failure mechanism provided by a random heterogeneous mesh and the homogenized approach.

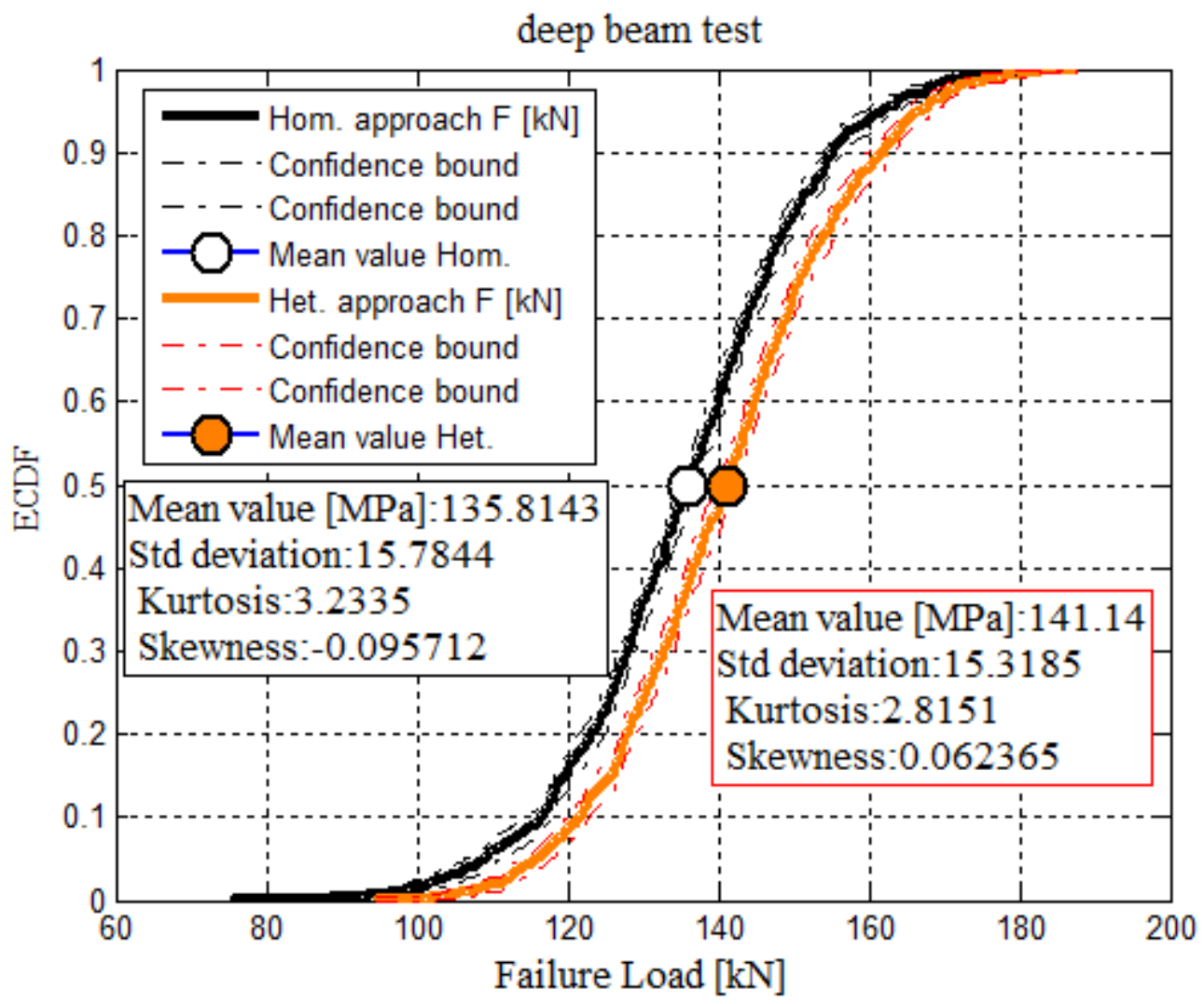

Figure 22: Deep beam example. ECDF of the failure load provide through a direct heterogeneous approach and homogenized limit analysis simulations. 

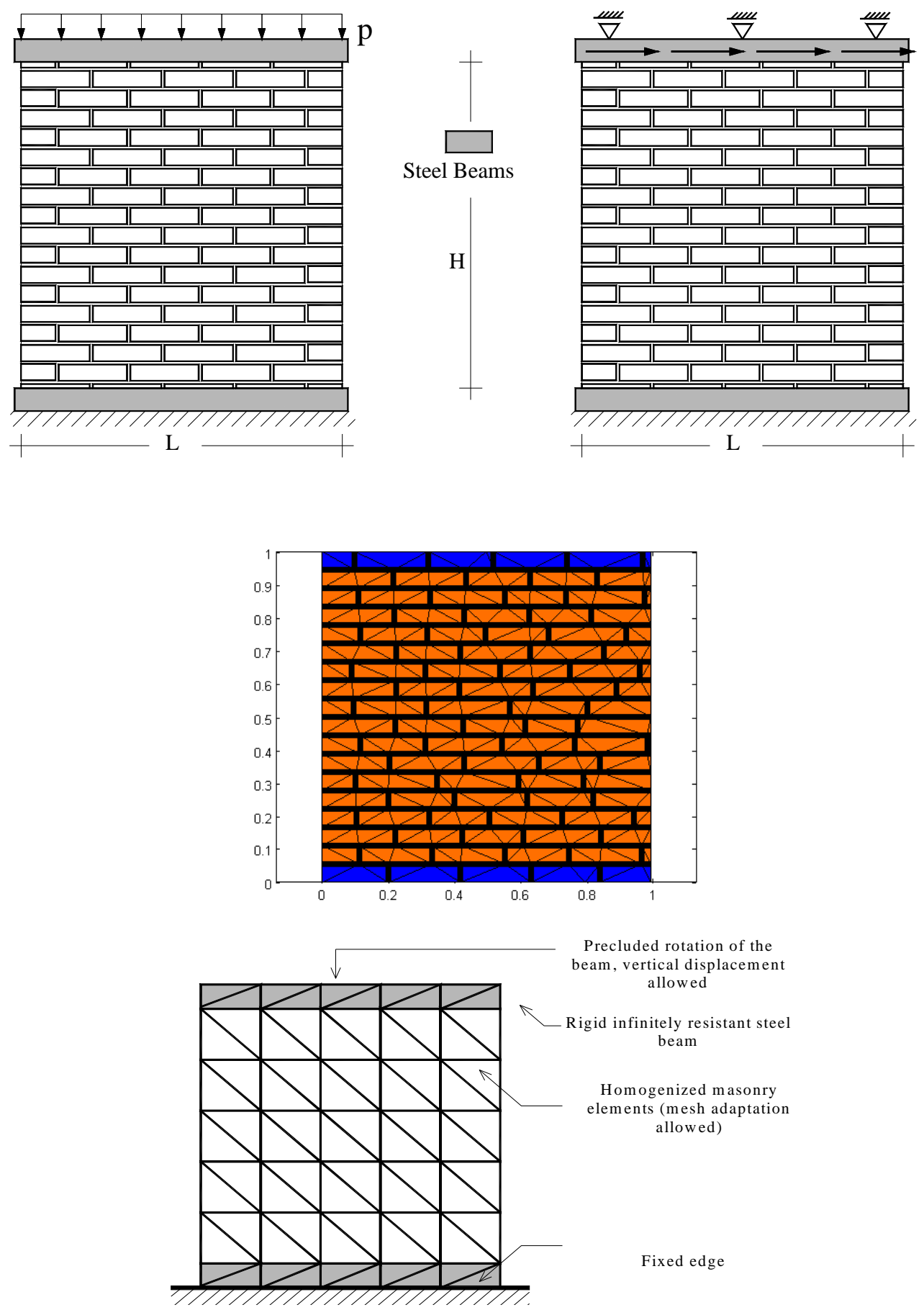

Figure 23: Shear wall example. Geometry, loading and constraints condition, typical heterogeneous and homogenized mesh used. 

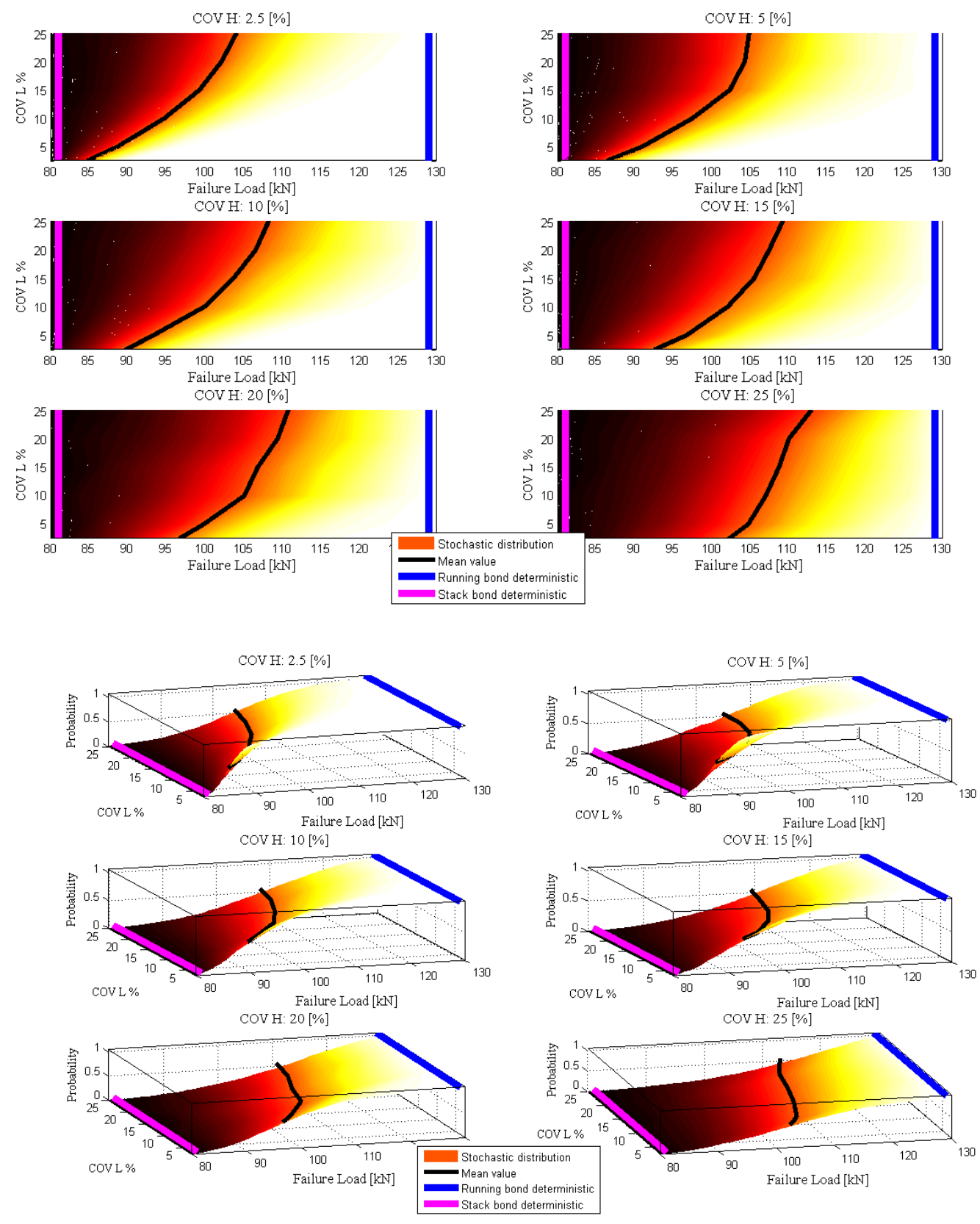

Figure 24: Shear wall example. Failure load distribution at different COVs of blocks length and height (Case I). 

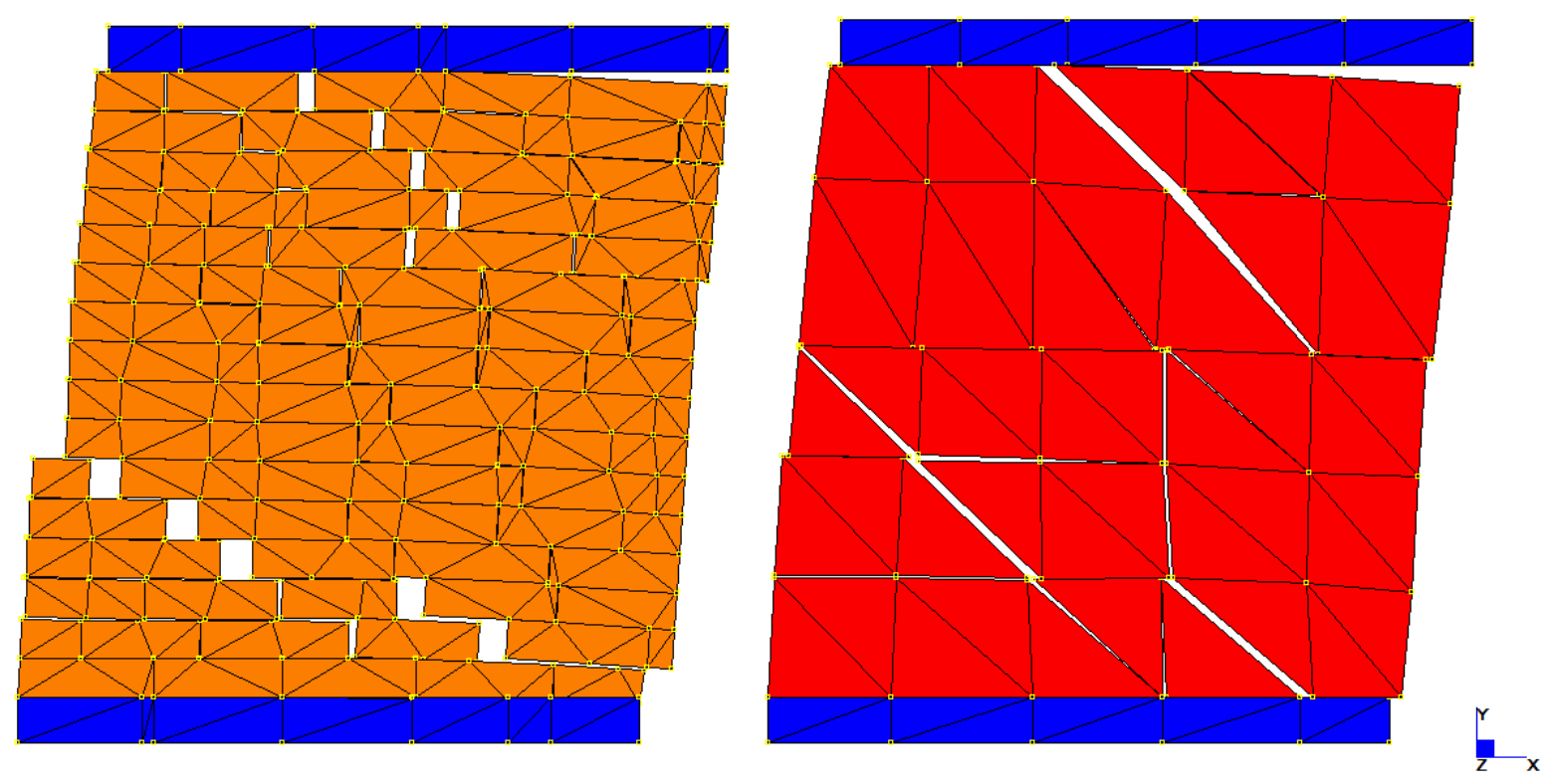

Figure 25: Shear wall example. Failure mechanism provided by a random heterogeneous mesh and the homogenized approach.

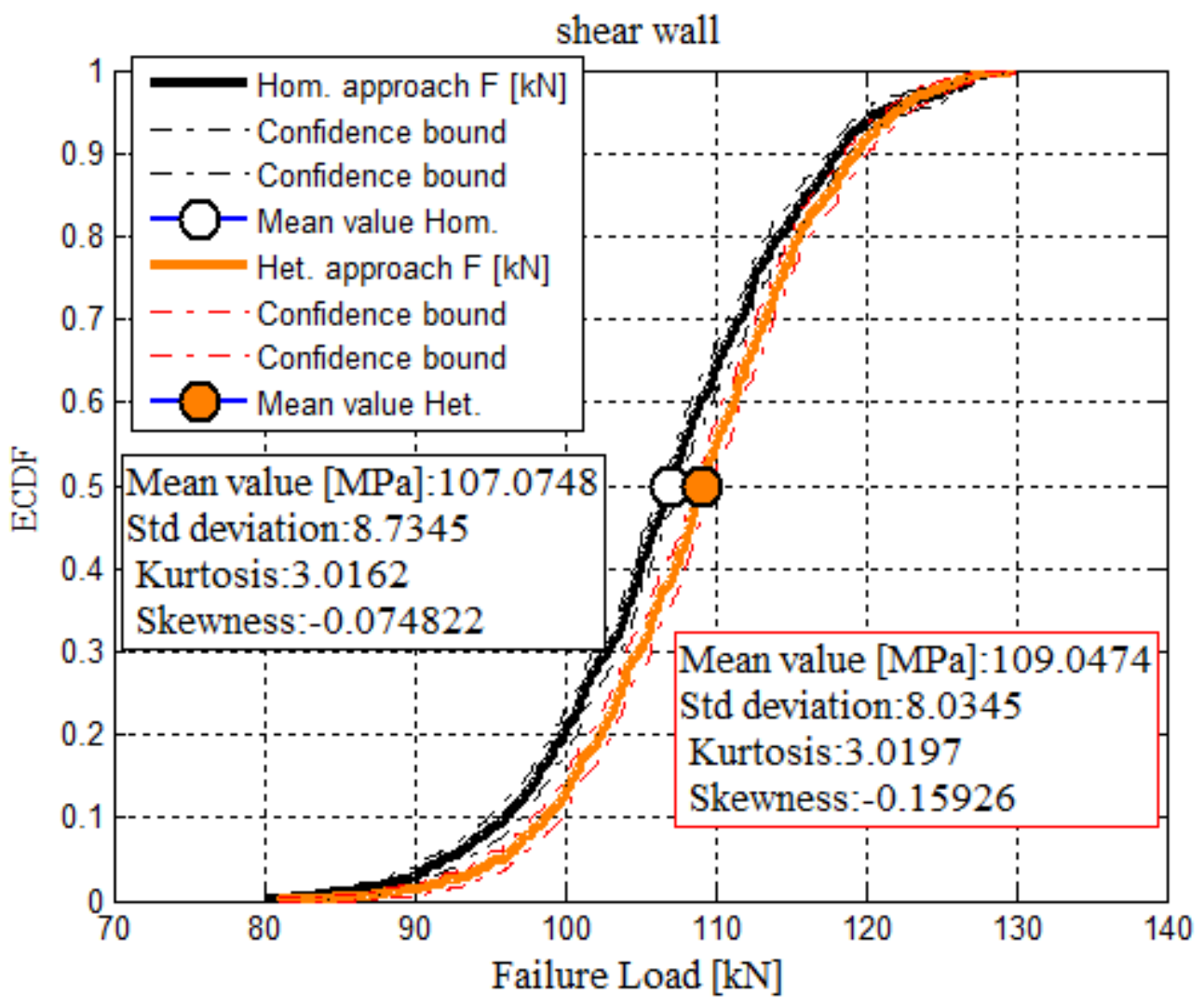

Figure 26: Shear wall example. ECDF of the failure load provide through a direct heterogeneous approach a homogenized limit analysis simulations. 


\section{Tables}

Table I: Representative element of volume level. Mechanical characteristics assumed for mortar joints reduced to interfaces $\left(f_{t}:\right.$ tension cut-off, $c$ : cohesion, $\Phi$ : friction angle, $f_{c}:$ compressive strength, $\Phi_{2}$ : shape of the linearized compressive cap).

\begin{tabular}{|c|c|c|c|c|}
\hline$f_{t} \mathrm{MPa}$ & $c$ & $\Phi$ & $f_{c} \mathrm{MPa}$ & $\Phi_{2}$ \\
\hline 0.05 & $1.4 f_{t}$ & $37^{\circ}$ & 1.5 & $60^{\circ}$ \\
\hline
\end{tabular}

Table II: Structural level, deep beam wall. Mechanical characteristics assumed for mortar joints reduced to interfaces $\left(f_{t}\right.$ : tension cut-off, $c$ : cohesion, $\Phi$ : friction angle, $f_{c}$ : compressive strength, $\Phi_{2}$ : shape of the linearized compressive cap).

\begin{tabular}{|c|c|c|c|c|}
\hline$f_{t} \mathrm{MPa}$ & $c$ & $\Phi$ & $f_{c} \mathrm{MPa}$ & $\Phi_{2}$ \\
\hline 0.29 & $1.4 f_{t}$ & $30^{\circ}$ & 8.6 & $60^{\circ}$ \\
\hline
\end{tabular}

Table III: Structural level, shear wall. Mechanical characteristics assumed for mortar joints reduced to interfaces $\left(f_{t}\right.$ : tension cut-off, $c$ : cohesion, $\Phi$ : friction angle, $f_{c}$ : compressive strength, $\Phi_{2}$ : shape of the linearized compressive cap).

\begin{tabular}{|c|c|c|c|c|}
\hline$f_{t} \mathrm{MPa}$ & $c$ & $\Phi$ & $f_{c} \mathrm{MPa}$ & $\Phi_{2}$ \\
\hline 0.16 & $1.4 f_{t}$ & $37^{\circ}$ & 11.5 & $30^{\circ}$ \\
\hline
\end{tabular}

